\title{
Optimal distributed control of a diffuse interface model of tumor growth*
}

\author{
Pierluigi Colli ${ }^{\dagger}$, Gianni Gilardi ${ }^{\ddagger}$, Elisabetta Rocca ${ }^{\S}$ \\ and Jürgen Sprekels
}

\begin{abstract}
In this paper, a distributed optimal control problem is studied for a diffuse interface model of tumor growth which was proposed by Hawkins-Daruud et al. in [25]. The model consists of a Cahn-Hilliard equation for the tumor cell fraction $\varphi$ coupled to a reaction-diffusion equation for a function $\sigma$ representing the nutrientrich extracellular water volume fraction. The distributed control $u$ monitors as a right-hand side the equation for $\sigma$ and can be interpreted as a nutrient supply or a medication, while the cost function, which is of standard tracking type, is meant to keep the tumor cell fraction under control during the evolution. We show that the control-to-state operator is Fréchet differentiable between appropriate Banach spaces and derive the first-order necessary optimality conditions in terms of a variational inequality involving the adjoint state variables.
\end{abstract}

Key words: Distributed optimal control, first-order necessary optimality conditions, tumor growth, reaction-diffusion equations, Cahn-Hilliard equation.

AMS (MOS) subject classification: 35K61,49J20, 49K20, 92C50.

*The financial support of the FP7-IDEAS-ERC-StG \#256872 (EntroPhase) is gratefully acknowledged. The paper also benefits from the support of the MIUR-PRIN Grant 2010A2TFX2 "Calculus of Variations" for PC and GG, and the GNAMPA (Gruppo Nazionale per l'Analisi Matematica, la Probabilità e le loro Applicazioni) of INdAM (Istituto Nazionale di Alta Matematica) for PC, GG and ER.

†Dipartimento di Matematica "F. Casorati," Università di Pavia, and IMATI-C.N.R., Via Ferrata 1, I-27100 Pavia, Italy (pierluigi.colli@unipv.it).

‡Dipartimento di Matematica "F. Casorati," Università di Pavia, and IMATI-C.N.R., Via Ferrata 1, I-27100 Pavia, Italy (gianni.gilardi@unipv.it).

$\S$ Weierstrass Institute for Applied Analysis and Stochastics, Mohrenstrasse 39, D-10117 Berlin, Germany (rocca@wias-berlin.de), and Dipartimento di Matematica, Università di Milano, Via Saldini 50, I-20133 Milano, Italy (elisabetta.rocca@unimi.it).

"Weierstrass Institute for Applied Analysis and Stochastics, Mohrenstrasse 39, D-10117 Berlin, Germany (sprekels@wias-berlin.de), and Institut für Mathematik der Humboldt-Universität zu Berlin, Unter den Linden 6, D-10099 Berlin, Germany. 


\section{Introduction}

Let $\Omega \subset \mathbb{R}^{3}$ be an open bounded and connected set with a smooth boundary $\partial \Omega$, and let $\mathbf{n}$ denote the outward unit normal to $\partial \Omega$. Moreover, let a fixed final time $T>0$ be given and $Q:=\Omega \times(0, T), \Sigma:=\partial \Omega \times(0, T)$. We investigate in this paper the following distributed optimal control problem:

(CP) Minimize the cost functional

$$
\begin{aligned}
\mathcal{J}(\varphi, u)= & \frac{\beta_{Q}}{2} \int_{0}^{T} \int_{\Omega}\left|\varphi-\varphi_{Q}\right|^{2} \mathrm{~d} x \mathrm{~d} t+\frac{\beta_{\Omega}}{2} \int_{\Omega}\left|\varphi(T)-\varphi_{\Omega}\right|^{2} \mathrm{~d} x \\
& +\frac{\beta_{u}}{2} \int_{0}^{T} \int_{\Omega}|u|^{2} \mathrm{~d} x \mathrm{~d} t
\end{aligned}
$$

subject to the control constraint

$$
u \in \mathcal{U}_{\mathrm{ad}}:=\left\{u \in L^{\infty}(Q): u_{\min } \leq u \leq u_{\max } \text { a. e. in } Q\right\}
$$

and to the state system

$$
\begin{aligned}
& \varphi_{t}-\Delta \mu=P(\varphi)(\sigma-\delta \mu) \quad \text { in } Q, \\
& \mu=-\Delta \varphi+F^{\prime}(\varphi) \quad \text { in } Q, \\
& \sigma_{t}-\Delta \sigma=-P(\varphi)(\sigma-\delta \mu)+u \text { in } Q, \\
& \partial_{\mathbf{n}} \varphi=\partial_{\mathbf{n}} \mu=\partial_{\mathbf{n}} \sigma=0 \quad \text { on } \Sigma, \\
& \varphi(0)=\varphi_{0}, \quad \sigma(0)=\sigma_{0} \quad \text { in } \Omega .
\end{aligned}
$$

The quantities occurring in the above expressions have the following meaning: $\beta_{Q}$, $\beta_{\Omega}, \beta_{u}$ are nonnegative constants, $\delta>0$ is a constant, $\varphi_{Q} \in L^{2}(Q), \varphi_{\Omega} \in L^{2}(\Omega)$, $u_{\min } \in L^{\infty}(Q), u_{\max } \in L^{\infty}(Q)$ are given functions such that $u_{\min } \leq u_{\max }$ almost everywhere in $Q, F$ and $P$ are given nonlinearities, $\partial_{\mathbf{n}}$ denotes the derivative in the direction of the outward unit normal $\mathbf{n}$, and $\varphi_{0}, \sigma_{0}$ are given initial data. In the following, we will always assume that $\delta=1$, which has no bearing on the mathematical analysis.

The state equations (1.3)-(1.7) constitute an approximation to a model for the dynamics of tumor growth due to [25] (see also [26, 44]) in which the velocities are set to zero and the only state variables considered are the tumor cell fraction $\varphi$ and the nutrient-rich extracellular water fraction $\sigma$. Typically, the function $F$ occurring in the chemical potential $\mu$ is a double-well potential, and $P$ denotes a suitable proliferation function, which is in general a nonnegative and regular function of $\varphi$.

Altogether, the optimal control problem $(\mathbf{C P})$ can be interpreted as the search for a strategy how to apply a control $u$ (which may represent the supply of a nutrient (see [4]), or even a drug in a chemotherapy) properly in order that

(i) the integral over the full space-time domain of the squared amount of nutrient or drug supplied (which is restricted by the control constraints) does not inflict any 
harm on the patient (which is expressed by the presence of the third summand in the cost functional);

(ii) a desired evolution and final distribution of the tumor cells (which is expressed by the target functions $\varphi_{Q}$ and $\varphi_{\Omega}$ ) is realized in the best possible way.

The ratios $\beta_{Q} / \beta_{u}$ and $\beta_{\Omega} / \beta_{u}$ indicate which importance the conflicting targets 'avoid unnecessary harm to the patient' and 'quality of the approximation of $\varphi_{Q}, \varphi_{\Omega}$ ' are given in the strategy. We remark that in practice it would be safer for the patient (and thus more desirable) to approximate the target functions rather in the $L^{\infty}$ sense than in the $L^{2}$ sense; however, in view of the analytical difficulties that are inherent in the state system, this presently seems to be out of reach. Of course, other integral terms depending on $\sigma$ and analogous to the ones acting on $\varphi$ could be added to the control functional, and it is our opinion that such a modified functional may be tractable from a mathematical point of view. However, since the problem is already quite involved and it is not clear whether the modification is really worth to be considered for applications, we prefer not to include the extra terms in the cost functional.

The mathematical modeling of tumor growth dynamics has drawn much attention in the past decade (cf., e.g., [1, 15, 33, 37]). In particular, models based on continuum mixture theory have been derived (see [5, 16, 18, 24, 36, 43]), which usually lead to Cahn-Hilliard systems involving transport and reaction terms that govern various types of concentrations, where, in particular, the reaction terms depend on the nutrient concentration.

While there exist quite a number of numerical simulations of diffuse-interface models of tumor growth (cf., e.g., [15, Chap. 8], [16, 25, 43, 44], and the references given there), there are still only a few contributions to the mathematical analysis of the models. The first contributions in this direction dealt with the case where the nutrient is neglected, which then leads to the so-called Cahn-Hilliard-Hele-Shaw system (see $[3,31,34,41,42])$. Only very recently, in the paper [17], the authors proved existence of weak solutions and some rigorous sharp interface limit for a model introduced in [6] (cf. also [15, 16, 18, 33, 43]), where both velocities (satisfying a Darcy law with Korteweg term) and multispecies tumor fractions, as well as the nutrient evolutions are taken into account. Let us also quote the paper [23], where a new model for tumor growth including different densities is introduced and a formal sharp interface limit is performed; moreover, the well-posedness of the related diffuse interface model is discussed in [22]. Finally, in the contribution [19], the system (1.3)-(1.7), which constitutes an approximation of the model introduced in [25], was rigorously analyzed concerning well-posedness, regularity, and asymptotic behavior. We also refer to the recent papers [9 11], in which various 'viscous' approximations of the state system have been studied analytically.

In this paper, we focus on the control aspect. While there exist many contributions concerning the well-posedness of various types of Cahn-Hilliard systems, only a few deal with their optimal control. In this connection, we mention the papers [12, 13, 28, 40, which deal with zero Neumann boundary conditions like (1.6), while in the recent papers [7,8, 13, 14] dynamic boundary conditions have been studied. A num- 
ber of papers also investigates optimal control problems for convective Cahn-Hilliard systems (cf. [38, 45, 46]) and Cahn-Hilliard-Navier-Stokes systems (cf. [20, 27, 29, 30]). Regarding the problem of optimal control in tumor growth models, we can quote the papers [32], where the problem of minimizing the volume of tumor under isoperimetric contraints is considered, and [2], where an advection-reaction-diffusion system for leukemia development is studied. However, to the authors' best knowledge, optimal control problems for the system (1.3)-(1.7) have never been studied before.

Indeed, the main mathematical difficulties are related to the proofs of suitable stability estimates of higher order (with respect to the ones already present in [19]), which are necessary in order to prove the differentiability (in suitable spaces) of the control-to-state mapping. The presence of the two nonlinearities $F$ and $P$ is indeed the main challenge in the analysis. Moreover, due to the dependence on the $L^{2}(\Omega)-$ target $\varphi_{\Omega}$ in the final condition for the variable $p$, which is related to the tumor phase $\varphi$, we only get existence for the adjoint system in the sense that the $p$-equation has to be intended in a weak form, mainly in the dual of the Sobolev space $H^{2}(\Omega)$ (cf. Section 4 for further comments on this subject). Let us finally point out that, with a view to applications, it would be worth analyzing the case of an $L^{\infty}$-type control functional rather than the $L^{2}$-one tackled here; however, this would bring further difficulties in solving the adjoint system in which measures would occur on the righthand sides of the equations. Hence, since the present contribution is the first one on the control theory for diffuse models of tumor growth, we prefer to start with the $L^{2}$ - control function $\mathcal{J}$ in (1.1).

Plan of the paper. The paper is organized as follows: in Section 2, we formulate the general hypotheses and improve known results regarding the well-posedness and regularity of the state system (1.3)-(1.7) (Theorem 2.1). We also prove a continuous dependence result (Theorem 2.2) which is needed for the analysis of the control problem. In Section 3, we study the differentiability properties of the control-to-state operator. The main results of this paper concerning existence and first-order necessary optimality conditions for the optimal control problem (CP) are shown in Section 4.

Throughout this paper, for a (real) Banach space $X$ we denote by $\|\cdot\|_{X}$ its norm, by $X^{\prime}$ its dual space, and by $\langle\cdot, \cdot\rangle_{X}$ the dual pairing between $X^{\prime}$ and $X$. If $X$ is an inner product space, then the inner product is denoted by $(\cdot, \cdot)_{X}$. The only exception from this convention is given by the $L^{p}$ spaces, $1 \leq p \leq \infty$, for which we use the abbreviating notation $\|\cdot\|_{p}$ for the norms in both $L^{p}(\Omega)$ and $L^{p}(Q)$. Moreover, we will use the notations

$$
H:=L^{2}(\Omega), \quad V:=H^{1}(\Omega), \quad W:=\left\{w \in H^{2}(\Omega): \partial_{\mathbf{n}} w=0 \text { a. e. on } \partial \Omega\right\} .
$$

We have the dense and continuous embeddings $W \subset V \subset H \cong H^{\prime} \subset V^{\prime} \subset W^{\prime}$, where $\langle u, v\rangle_{V}=(u, v)_{H}$ and $\langle u, w\rangle_{W}=(u, w)_{H}$ for all $u \in H, v \in V$, and $w \in W$.

During the course of this paper, we will make repeated use of Young's inequality

$$
a b \leq \delta a^{2}+\frac{1}{4 \delta} b^{2} \quad \text { for all } a, b \in \mathbb{R} \text { and } \delta>0,
$$


as well as of the fact that for three dimensions of space and smooth domains the embeddings $V \subset L^{p}(\Omega), 1 \leq p \leq 6$, and $H^{2}(\Omega) \subset C^{0}(\bar{\Omega})$ are continuous and (in the first case only for $1 \leq p<6$ ) compact. In particular, there are positive constants $\widetilde{K}_{i}$, $i=1,2,3$, which depend only on the domain $\Omega$, such that

$$
\begin{aligned}
\|v\|_{6} & \leq \widetilde{K}_{1}\|v\|_{V} \quad \forall v \in V, \\
\|v w\|_{H} & \leq\|v\|_{6}\|w\|_{3} \leq \widetilde{K}_{2}\|v\|_{V}\|w\|_{V} \quad \forall v, w \in V, \\
\|v\|_{L^{\infty}(\Omega)} & \leq \widetilde{K}_{3}\|v\|_{H^{2}(\Omega)} \quad \forall v \in H^{2}(\Omega) .
\end{aligned}
$$

Moreover, we have

$$
\|v w\|_{V^{\prime}} \leq\|v\|_{W^{1, \infty}(\Omega)}\|w\|_{V^{\prime}}, \quad \forall v \in W^{1, \infty}(\Omega), \quad \forall w \in V^{\prime} .
$$

Finally, we recall that for smooth and bounded three-dimensional domains there holds the special Gagliardo-Nirenberg inequality (cf. [35, p. 125])

$$
\|v\|_{3} \leq \widetilde{K}_{4}\left(\|v\|_{H}^{1 / 2}\|v\|_{V}^{1 / 2}+\|v\|_{H}\right) \quad \forall v \in V
$$

where the positive constant $\widetilde{K}_{4}$ depends only on $\Omega$.

\section{General assumptions and preliminary results on the state system}

In the following, we study the state system (1.3)-(1.7). Since it will be convenient to rewrite various partial differential equations in this paper as abstract equations in the framework of the Hilbert triple $\left(V, H, V^{\prime}\right)$, we introduce the Riesz isomorphism $A: V \rightarrow V^{\prime}$ associated with the standard scalar product of $V$, that is,

$$
\langle A u, v\rangle_{V}=(u, v)_{V}=\int_{\Omega}(\nabla u \cdot \nabla v+u v) \mathrm{d} x \quad \text { for } u, v \in V .
$$

We note that the restriction of $A$ to $W$, which is given by $A u=-\Delta u+u$, for $u \in W$, is an isomorphism from $W$ onto $H$. Moreover, the linear operator $A$ can be continuously extended to a linear mapping from $H$ into $W^{\prime}$, where $\langle A u, v\rangle_{W}=(u, A v)_{H}$ for all $u \in H$ and $v \in W$. We also remark that, for some positive constant $\widetilde{K}_{5}$ which depends only on $\Omega$, we have

$$
\begin{aligned}
\left\langle A u, A^{-1} v^{*}\right\rangle_{V} & =\left\langle v^{*}, u\right\rangle_{V} \quad \text { for all } u \in V \text { and } v^{*} \in V^{\prime}, \\
\left\langle u^{*}, A^{-1} v^{*}\right\rangle_{V} & =\left(u^{*}, v^{*}\right)_{V^{\prime}} \quad \text { for all } u^{*}, v^{*} \in V^{\prime}, \\
\left\|A^{-1} u^{*}\right\|_{V^{\prime}} & \leq \widetilde{K}_{5}\left\|u^{*}\right\|_{V^{\prime}} \quad \text { for all } u^{*} \in V^{\prime},
\end{aligned}
$$

where $(\cdot, \cdot)_{V^{\prime}}$ is the dual scalar product in $V^{\prime}$ associated with the standard one in $V$. We also have, for every $v^{*} \in H^{1}\left(0, T ; V^{\prime}\right)$,

$$
\frac{d}{d t}\left\|v^{*}(t)\right\|_{V^{\prime}}^{2}=2\left\langle\partial_{t} v^{*}(t), A^{-1} v^{*}(t)\right\rangle_{V} \quad \text { for almost every } t \in(0, T) .
$$


We make for the remainder of this paper the following general assumptions on the data of the control problem $(\mathbf{C P})$ :

(H1) $\beta_{Q}, \beta_{\Omega}, \beta_{u}$ are nonnegative but not all zero.

(H2) $\varphi_{Q} \in L^{2}(Q), \varphi_{\Omega} \in L^{2}(\Omega), u_{\min } \in L^{\infty}(Q), u_{\max } \in L^{\infty}(Q)$, with $u_{\min } \leq u_{\max }$ a. e. in $Q$.

(H3) $\varphi_{0} \in H^{3}(\Omega), \sigma_{0} \in H^{1}(\Omega)$.

(H4) $P \in C_{\text {loc }}^{1,1}(\mathbb{R})$ is nonnegative and satisfies, for almost every $s \in \mathbb{R}$,

$$
\left|P^{\prime}(s)\right| \leq \alpha_{1}\left(1+|s|^{q-1}\right) \text {, with some } \alpha_{1}>0 \text { and some } q \in[1,4] .
$$

(H5) $F \in C^{4}(\mathbb{R})$ can be written in the form $F=F_{0}+F_{1}$, where $F_{0}, F_{1} \in C^{4}(\mathbb{R})$, and where there are constants $\alpha_{i}>0,2 \leq i \leq 6$, and $\rho \in[2,6)$ such that

$$
\begin{aligned}
& \left|F_{1}^{\prime \prime}(s)\right| \leq \alpha_{2} \quad \forall s \in \mathbb{R}, \\
& \alpha_{3}\left(1+|s|^{\rho-2}\right) \leq F_{0}^{\prime \prime}(s) \leq \alpha_{4}\left(1+|s|^{\rho-2}\right) \quad \forall s \in \mathbb{R}, \\
& F(s) \geq \alpha_{5}|s|-\alpha_{6} \quad \forall s \in \mathbb{R} .
\end{aligned}
$$

The conditions $(\mathbf{H 3})-(\mathbf{H} 5)$ originate from the paper [19], where they were postulated to guarantee the validity of some well-posedness results. We remark that not all of them are needed for some of the results proved in [19]; however, they are indispensable for the analysis of the control problem (CP) on which we focus in this paper. The following hypothesis is rather a denotation than an assumption:

(H6) $\mathcal{U}_{R}$ is an open set in $L^{2}(Q)$ such that $\mathcal{U}_{\text {ad }} \subset \mathcal{U}_{R}$ and $\|u\|_{L^{2}(Q)} \leq R$ for all $u \in \mathcal{U}_{R}$.

We have the following well-posedness result for the state system (1.3)-(1.7).

THEOREM 2.1 Suppose that the hypotheses $(\mathbf{H 1})-(\mathbf{H 6})$ are fulfilled. Then the follwing results hold true:

(i) For every $u \in \mathcal{U}_{R}$, the state system (1.3)-(1.7) has a unique strong solution triple $(\varphi, \mu, \sigma)$ such that

$$
\begin{aligned}
& \varphi \in H^{1}(0, T ; V) \cap L^{\infty}\left(0, T ; W \cap H^{3}(\Omega)\right), \quad \Delta \varphi \in L^{2}(0, T ; W), \\
& \mu \in L^{\infty}(0, T ; V) \cap L^{2}(0, T ; W), \\
& \sigma \in H^{1}(0, T ; H) \cap C^{0}([0, T] ; V) \cap L^{2}(0, T ; W) .
\end{aligned}
$$

(ii) There is some constant $K_{1}^{*}>0$, which depends only on $R$ and the data of the system, such that for every $u \in \mathcal{U}_{R}$ the associated strong solution $(\varphi, \mu, \sigma)$ to (1.3)(1.7) satisfies

$$
\begin{aligned}
& \|\varphi\|_{H^{1}(0, T ; V) \cap L^{\infty}\left(0, T ; H^{3}(\Omega)\right)}+\|\Delta \varphi\|_{L^{2}\left(0, T ; H^{2}(\Omega)\right)}+\|\mu\|_{L^{\infty}(0, T ; V) \cap L^{2}\left(0, T ; H^{2}(\Omega)\right)} \\
& +\|\sigma\|_{H^{1}(0, T ; H) \cap C^{0}([0, T] ; V) \cap L^{2}\left(0, T ; H^{2}(\Omega)\right)} \leq K_{1}^{*} .
\end{aligned}
$$


(iii) There is some constant $K_{2}^{*}>0$, which depends only on $R$ and the data of the problem, such that the following holds true: whenever $u_{i} \in \mathcal{U}_{R}, i=1,2$, are given and $\left(\varphi_{i}, \mu_{i}, \sigma_{i}\right), i=1,2$, are the associated solutions to the state system, then we have, for every $t \in[0, T]$,

$$
\begin{aligned}
& \left\|\varphi_{1}(t)-\varphi_{2}(t)\right\|_{V^{\prime}}+\left\|\varphi_{1}-\varphi_{2}\right\|_{L^{2}(0, t ; V)}+\left\|\sigma_{1}(t)-\sigma_{2}(t)\right\|_{V^{\prime}} \\
& +\left\|\sigma_{1}-\sigma_{2}\right\|_{L^{2}(0, t ; H)} \leq K_{2}^{*}\left\|u_{1}-u_{2}\right\|_{L^{2}(0, t ; H)} .
\end{aligned}
$$

Proof: In the following, we denote by $C_{i}>0, i \in \mathbb{N}$, constants that depend only on $R$ and the data entering the state system. In [19, Thms. 1-3], it has been shown that the variational problem

$$
\begin{aligned}
& \int_{\Omega} \partial_{t} \varphi(t) v \mathrm{~d} x+\int_{\Omega} \nabla \mu(t) \cdot \nabla v \mathrm{~d} x=\int_{\Omega} P(\varphi(t))(\sigma(t)-\mu(t)) v \mathrm{~d} x \\
& \int_{\Omega} \mu(t) v \mathrm{~d} x=\int_{\Omega} \nabla \varphi(t) \cdot \nabla v \mathrm{~d} x+\int_{\Omega} F^{\prime}(\varphi(t)) v \mathrm{~d} x \\
& \int_{\Omega} \partial_{t} \sigma(t) v \mathrm{~d} x+\int_{\Omega} \nabla \sigma(t) \cdot \nabla v \mathrm{~d} x=\int_{\Omega}(u(t)-P(\varphi(t))(\sigma(t)-\mu(t))) v \mathrm{~d} x
\end{aligned}
$$

for all $v \in V$ and almost every $t \in(0, T)$, has in the homogeneous case $u \equiv 0$ a unique solution triple $(\varphi, \mu, \sigma)$ which satisfies the initial conditions (1.7) and has the regularity properties

$$
\begin{aligned}
& \varphi \in H^{1}(0, T ; V) \cap L^{\infty}\left(0, T ; H^{3}(\Omega)\right), \quad \mu \in L^{\infty}(0, T ; V), \\
& \sigma \in H^{1}(0, T ; H) \cap L^{\infty}(0, T ; V) .
\end{aligned}
$$

A closer inspection of the proofs of [19, Thms. 1-3] (in particular, Eq. (2.13) is nowhere differentiated with respect to time) reveals that only straightforward modifications are needed to show that the system (2.11)-(2.13), (1.7) has for every $u \in \mathcal{U}_{R}$ a unique solution triple $(\varphi, \mu, \sigma)$ which has the regularity properties (2.14) and satisfies, with some $C_{1}>0$,

$$
\|\varphi\|_{H^{1}(0, T ; V) \cap L^{\infty}\left(0, T ; H^{3}(\Omega)\right)}+\|\mu\|_{L^{\infty}(0, T ; V)}+\|\sigma\|_{H^{1}(0, T ; H) \cap L^{\infty}(0, T ; V)} \leq C_{1} .
$$

Now observe that (2.15) and (H4) imply that $u-P(\varphi)(\sigma-\mu)$ is bounded in $L^{2}(Q)$. Since $\sigma_{0} \in V$, parabolic regularity theory, applied to (2.13), yields $\sigma \in L^{2}(0, T ; W)$ and the bound for $\sigma$ stated in (2.9). Moreover, Eq. (2.11) is for almost every $t \in(0, T)$ the weak form of the elliptic problem

$$
-\Delta \mu(t)=P(\varphi(t))(\sigma(t)-\mu(t))-\partial_{t} \varphi(t) \quad \text { in } \Omega, \quad \partial_{\mathbf{n}} \mu(t)=0 \quad \text { on } \partial \Omega .
$$

By (H4) and (2.15), we have $P(\varphi)(\sigma-\mu)-\partial_{t} \varphi \in L^{2}(0, T ; H)$, whence we infer from elliptic regularity theory that $\mu(t) \in W$ for almost every $t \in(0, T)$, as well as $\|\mu\|_{L^{2}\left(0, T ; H^{2}(\Omega)\right)}$ is uniformly bounded. Moreover, we have $\partial_{\mathbf{n}}(\Delta \varphi)=-\partial_{\mathbf{n}} \mu+$ $F^{\prime \prime}(\varphi) \partial_{\mathbf{n}} \varphi=0$ almost everywhere on $\Sigma$, as well as

$$
\Delta^{2} \varphi=-\Delta \mu+F^{\prime \prime}(\varphi) \Delta \varphi+F^{\prime \prime \prime}(\varphi)|\nabla \varphi|^{2},
$$


where, due to (2.15) and (H5), the right-hand side is bounded in $L^{2}(Q)$. We thus have $\Delta \varphi \in L^{2}(0, T ; W)$ with bounded norm, and the assertions (i) and (ii) are proved. The last assertion (iii) can be shown in exactly the same way as the stability result shown in the proof of [19, Thm.2]. We therefore may skip the proof.

REMARK 1 Observe that standard embedding results (cf. [39, Sec. 8, Cor. 4]) imply that $H^{1}(0, T ; V) \cap L^{\infty}\left(0, T ; H^{2}(\Omega)\right)$ is continuously embedded in $C^{0}\left([0, T] ; H^{s}(\Omega)\right)$ for $0<s<2$. Consequently, $\varphi \in C^{0}(\bar{Q})$. Moreover, also owing to the continuity of the embeddings $V \subset L^{6}(\Omega)$ and $H^{2}(\Omega) \subset L^{\infty}(\Omega)$ in three dimensions of space, and invoking the general hypotheses $(\mathbf{H} 4)$ and $(\mathbf{H 5})$, we may without loss of generality state (possibly choosing a larger $K_{1}^{*}$ ) the following bounds:

$$
\begin{aligned}
& \|\varphi\|_{C^{0}(\bar{Q})}+\|\nabla \varphi\|_{L^{\infty}(Q)}+\max _{0 \leq i \leq 4}\left\|F^{(i)}(\varphi)\right\|_{C^{0}(\bar{Q})}+\max _{0 \leq i \leq 2}\left\|P^{(i)}(\varphi)\right\|_{L^{\infty}(Q)} \\
& +\|\Delta \varphi\|_{L^{\infty}\left(0, T ; L^{6}(\Omega)\right) \cap L^{2}\left(0, T ; L^{\infty}(\Omega)\right)}+\|\mu\|_{L^{\infty}\left(0, T ; L^{6}(\Omega)\right) \cap L^{2}\left(0, T ; L^{\infty}(\Omega)\right)} \\
& +\|\sigma\|_{L^{\infty}\left(0, T ; L^{6}(\Omega)\right) \cap L^{2}\left(0, T ; L^{\infty}(\Omega)\right)} \leq K_{1}^{*},
\end{aligned}
$$

for any solution $(\varphi, \mu, \sigma)$ corresponding to some $u \in \mathcal{U}_{R}$.

REMARK 2 A comparison argument yields that (by possibly choosing a larger $K_{2}^{*}$ ) the estimate

$$
\left\|\mu_{1}-\mu_{2}\right\|_{L^{2}\left(0, t ; V^{\prime}\right)} \leq K_{2}^{*}\left\|u_{1}-u_{2}\right\|_{L^{2}(0, t ; H)}
$$

holds true for every $t \in[0, T]$ with $\mu_{i}:=-\Delta \varphi_{i}+F^{\prime}\left(\varphi_{i}\right), i=1,2$. In particular, the control-to-state operator $\mathcal{S}, u \mapsto \mathcal{S}(u):=(\varphi, \mu, \sigma)$, is well defined and Lipschitz continuous as a mapping from $\mathcal{U}_{R} \subset L^{2}(Q)$ into the space

$$
\left(L^{\infty}\left(0, T, V^{\prime}\right) \cap L^{2}(0, T ; V)\right) \times L^{2}\left(0, T ; V^{\prime}\right) \times\left(L^{\infty}\left(0, T ; V^{\prime}\right) \cap L^{2}(0, T ; H)\right) .
$$

The stability results (2.10), (2.18) are not sufficient for studying the control problem $(\mathbf{C P})$. We thus begin our analysis by proving stronger stability estimates. We have the following result.

TheOREM 2.2 Suppose that the hypotheses $(\mathbf{H 1})-(\mathbf{H} 6)$ are fulfilled. Then there exists a constant $K_{3}^{*}>0$, which depends only on $R$ and the data of the system, such that the following holds true: whenever $u_{i} \in \mathcal{U}_{R}, i=1,2$, are given and $\left(\varphi_{i}, \mu_{i}, \sigma_{i}\right)$, $i=1,2$, are the associated solutions to the state system (1.3)-(1.7), then we have, for every $t \in[0, T]$,

$$
\begin{aligned}
& \left\|\varphi_{1}-\varphi_{2}\right\|_{H^{1}\left(0, t ; V^{\prime}\right) \cap L^{\infty}(0, t ; V) \cap L^{2}\left(0, t ; H^{3}(\Omega)\right)}+\left\|\mu_{1}-\mu_{2}\right\|_{L^{2}(0, t ; V)} \\
& +\left\|\sigma_{1}-\sigma_{2}\right\|_{H^{1}(0, t ; H) \cap C^{0}([0, t] ; V) \cap L^{2}\left(0, t ; H^{2}(\Omega)\right)} \leq K_{3}^{*}\left\|u_{1}-u_{2}\right\|_{L^{2}(0, t ; H)} .
\end{aligned}
$$

Proof: First, recall that the estimates (2.10) and (2.18) are valid. We again denote by $C_{i}>0, i \in \mathbb{N}$, constants that depend only on $R$ and the data entering the state 
system. Putting $\varphi:=\varphi_{1}-\varphi_{2}, \mu:=\mu_{1}-\mu_{2}, \sigma:=\sigma_{1}-\sigma_{2}$, and $u:=u_{1}-u_{2}$, we find the following identities:

$$
\begin{aligned}
& \partial_{t} \varphi-\Delta \mu=P\left(\varphi_{1}\right)(\sigma-\mu)+\left(P\left(\varphi_{1}\right)-P\left(\varphi_{2}\right)\right)\left(\sigma_{2}-\mu_{2}\right) \quad \text { a.e. in } Q, \\
& \mu=-\Delta \varphi+F^{\prime}\left(\varphi_{1}\right)-F^{\prime}\left(\varphi_{2}\right) \quad \text { a.e. in } Q, \\
& \partial_{t} \sigma-\Delta \sigma=u-P\left(\varphi_{1}\right)(\sigma-\mu)-\left(P\left(\varphi_{1}\right)-P\left(\varphi_{2}\right)\right)\left(\sigma_{2}-\mu_{2}\right) \quad \text { a.e. in } Q, \\
& \partial_{\mathbf{n}} \varphi=\partial_{\mathbf{n}} \mu=\partial_{\mathbf{n}} \sigma=0 \quad \text { a.e. on } \Sigma, \\
& \varphi(0)=\sigma(0)=0 \quad \text { a.e. in } \Omega .
\end{aligned}
$$

We now establish the asserted stability estimate (2.19) in a series of estimates in which we make repeated use of the global bounds (2.9) and (2.17) without further reference.

FiRst estimate: We multiply (2.20) by $\varphi$ and (2.21) by $\Delta \varphi$ and add the resulting equations to obtain that

$$
\begin{aligned}
\varphi \partial_{t} \varphi-\varphi \Delta \mu+\mu \Delta \varphi= & P\left(\varphi_{1}\right)(\sigma-\mu) \varphi+\left(P\left(\varphi_{1}\right)-P\left(\varphi_{2}\right)\right)\left(\sigma_{2}-\mu_{2}\right) \varphi \\
& -|\Delta \varphi|^{2}+\left(F^{\prime}\left(\varphi_{1}\right)-F^{\prime}\left(\varphi_{2}\right)\right) \Delta \varphi .
\end{aligned}
$$

Integration over $\Omega \times[0, t]$ (where $t \in[0, T]$ ) and by parts yield, using the mean value theorem, (H4), (H5), and Young's inequality, that

$$
\begin{aligned}
& \frac{1}{2}\|\varphi(t)\|_{H}^{2}+\frac{1}{2} \int_{0}^{t} \int_{\Omega}|\Delta \varphi|^{2} \mathrm{~d} x \mathrm{~d} s \\
& \leq I_{1}+C_{2} \int_{0}^{t}\left(\|\sigma(s)\|_{H}^{2}+\|\varphi(s)\|_{H}^{2}\right) \mathrm{d} s+C_{3} I_{2},
\end{aligned}
$$

where, owing to $(\mathbf{H} 4)$, (2.9), (2.10), (2.17), (2.18), and Hölder's inequality, we deduce that

$$
\begin{aligned}
I_{1} & :=\int_{0}^{t}\|\mu(s)\|_{V^{\prime}}\left\|P\left(\varphi_{1}(s)\right) \varphi(s)\right\|_{V} \mathrm{~d} s \\
& \leq \frac{1}{2} \int_{0}^{t}\|\mu(s)\|_{V^{\prime}}^{2} \mathrm{~d} s+\frac{1}{2} \int_{0}^{t}\left\|P\left(\varphi_{1}(s)\right) \varphi(s)\right\|_{V}^{2} \mathrm{~d} s \\
& \leq C_{4}\|u\|_{L^{2}(0, t ; H)}^{2}+\frac{1}{2} \int_{0}^{t} \int_{\Omega}\left(\left|P\left(\varphi_{1}\right) \varphi\right|^{2}+\left|P\left(\varphi_{1}\right) \nabla \varphi+\varphi P^{\prime}\left(\varphi_{1}\right) \nabla \varphi_{1}\right|^{2}\right) \mathrm{d} x \mathrm{~d} s \\
& \leq C_{4}\|u\|_{L^{2}(0, t ; H)}^{2}+C_{5} \int_{0}^{t}\|\varphi(s)\|_{V}^{2} \mathrm{~d} s \leq C_{6}\|u\|_{L^{2}(0, t ; H)}^{2}, \\
I_{2} & :=\int_{0}^{t} \int_{\Omega}|\varphi|^{2}\left(\left|\sigma_{2}\right|+\left|\mu_{2}\right|\right) \mathrm{d} x \mathrm{~d} s \leq \int_{0}^{t}\left(\left\|\sigma_{2}(s)\right\|_{6}+\left\|\mu_{2}(s)\right\|_{6}\right)\|\varphi(s)\|_{12 / 5}^{2} \mathrm{~d} s \\
& \leq C_{7} \int_{0}^{t}\|\varphi(s)\|_{V}^{2} \mathrm{~d} s \leq C_{8}\|u\|_{L^{2}(0, t ; H)}^{2} .
\end{aligned}
$$


Combining the above inequalities, using again (2.10), and invoking well-known elliptic regularity results, we infer the estimates

$$
\begin{aligned}
& \|\varphi\|_{L^{\infty}(0, t ; H) \cap L^{2}\left(0, t ; H^{2}(\Omega)\right)} \leq C_{9}\|u\|_{L^{2}(0, t ; H)} \text { for all } t \in[0, T] \\
& \|\mu\|_{L^{2}(0, t ; H)} \leq C_{10}\|u\|_{L^{2}(0, t ; H)} \quad \text { for all } t \in[0, T]
\end{aligned}
$$

where the second inequality follows from a comparison in (2.21), by applying once more the mean value theorem and (H5).

SECOnd Estimate: We now test (2.22) by $\partial_{t} \sigma$ and use Young's inequality and the mean value theorem along with $(\mathbf{H} 4)$ to find that, for all $t \in[0, T]$,

$$
\begin{aligned}
& \int_{0}^{t} \int_{\Omega}\left|\partial_{t} \sigma\right|^{2} \mathrm{~d} x \mathrm{~d} s+\frac{1}{2}\|\nabla \sigma(t)\|_{H}^{2} \\
& =\int_{0}^{t} \int_{\Omega} \partial_{t} \sigma\left[u-P\left(\varphi_{1}\right)(\sigma-\mu)-\left(P\left(\varphi_{1}\right)-P\left(\varphi_{2}\right)\right)\left(\sigma_{2}-\mu_{2}\right)\right] \mathrm{d} x \mathrm{~d} s \\
& \leq \frac{1}{4} \int_{0}^{t} \int_{\Omega}\left|\partial_{t} \sigma\right|^{2} \mathrm{~d} x \mathrm{~d} s+C_{11} \int_{0}^{t} \int_{\Omega}\left(u^{2}+\sigma^{2}+\mu^{2}\right) \mathrm{d} x \mathrm{~d} s+I,
\end{aligned}
$$

where, using Hölder's and Young's inequalities, as well as (2.17), we infer that

$$
\begin{aligned}
I & :=\int_{0}^{t} \int_{\Omega}\left|\partial_{t} \sigma\right|\left|P\left(\varphi_{1}\right)-P\left(\varphi_{2}\right)\right|\left(\left|\sigma_{2}\right|+\left|\mu_{2}\right|\right) \mathrm{d} x \mathrm{~d} s \\
& \leq C_{12} \int_{0}^{t}\left\|\partial_{t} \sigma(s)\right\|_{2}\|\varphi(s)\|_{3}\left(\left\|\sigma_{2}(s)\right\|_{6}+\left\|\mu_{2}(s)\right\|_{6}\right) \mathrm{d} s \\
& \leq \frac{1}{4} \int_{0}^{t} \int_{\Omega}\left|\partial_{t} \sigma\right|^{2} \mathrm{~d} x \mathrm{~d} s+C_{13} \int_{0}^{t}\|\varphi(s)\|_{V}^{2} \mathrm{~d} s .
\end{aligned}
$$

In view of (2.10) and (2.27), we thus obtain that

$$
\|\sigma\|_{H^{1}(0, t ; H) \cap L^{\infty}(0, t ; V)} \leq C_{14}\|u\|_{L^{2}(0, t ; H)} \text { for all } t \in[0, T]
$$

whence, by comparison in (2.22), and applying once more the mean value theorem together with $(\mathbf{H} 4)$, also the bound

$$
\|\sigma\|_{L^{2}\left(0, t ; H^{2}(\Omega)\right)} \leq C_{15}\|u\|_{L^{2}(0, t ; H)} \quad \text { for all } t \in[0, T]
$$

follows from the estimate of $\|\Delta \sigma\|_{L^{2}(0, t ; H)}$ and the elliptic regularity theory.

Third Estimate: Next, we insert $\mu$, given by (2.21), in (2.20) to find that

$$
\begin{aligned}
\partial_{t} \varphi+\Delta^{2} \varphi= & P\left(\varphi_{1}\right)(\sigma-\mu)+\left(P\left(\varphi_{1}\right)-P\left(\varphi_{2}\right)\right)\left(\sigma_{2}-\mu_{2}\right)+F^{\prime \prime}\left(\varphi_{1}\right) \Delta \varphi \\
& +\left(F^{\prime \prime}\left(\varphi_{1}\right)-F^{\prime \prime}\left(\varphi_{2}\right)\right) \Delta \varphi_{2}+F^{\prime \prime \prime}\left(\varphi_{1}\right)\left(\nabla \varphi_{1}+\nabla \varphi_{2}\right) \cdot \nabla \varphi \\
& +\left(F^{\prime \prime \prime}\left(\varphi_{1}\right)-F^{\prime \prime \prime}\left(\varphi_{2}\right)\right)\left|\nabla \varphi_{2}\right|^{2} .
\end{aligned}
$$


Testing this identity by $-\Delta \varphi \in L^{2}(0, T ; W)$, and using the integrations by parts, Young's inequality, the mean value theorem, (H4), (H5), and (2.17), we find that for any $t \in[0, T]$ we have

$$
\begin{aligned}
& \frac{1}{2}\|\nabla \varphi(t)\|_{H}^{2}+\int_{0}^{t} \int_{\Omega}|\nabla \Delta \varphi|^{2} \mathrm{~d} x \mathrm{~d} s \\
& \leq C_{16} \int_{0}^{t} \int_{\Omega}|\varphi|\left(\left|\sigma_{2}\right|+\left|\mu_{2}\right|+\left|\Delta \varphi_{2}\right|\right)|\Delta \varphi| \mathrm{d} x \mathrm{~d} s \\
& \quad+C_{17} \int_{0}^{t} \int_{\Omega}\left(|\varphi|^{2}+|\nabla \varphi|^{2}+|\Delta \varphi|^{2}+|\sigma|^{2}+|\mu|^{2}\right) \mathrm{d} x \mathrm{~d} s .
\end{aligned}
$$

The first integral on the right-hand side, which we denote by $I$, can be estimated as follows:

$$
\begin{aligned}
I & \leq \int_{0}^{t}\|\varphi(s)\|_{3}\left(\left\|\sigma_{2}(s)\right\|_{6}+\left\|\mu_{2}(s)\right\|_{6}+\left\|\Delta \varphi_{2}(s)\right\|_{6}\right)\|\Delta \varphi(s)\|_{2} \mathrm{~d} s \\
& \leq C_{18} \int_{0}^{t}\left(\|\varphi(s)\|_{V}^{2}+\|\Delta \varphi(s)\|_{H}^{2}\right) \mathrm{d} s \leq C_{19}\|u\|_{L^{2}(0, t ; H)}^{2},
\end{aligned}
$$

where the last inequality follows from (2.10) and (2.26). In conclusion, using once more (2.26) and (2.10) in order to bound the second integral in (2.32), we have the estimate

$$
\|\varphi\|_{L^{\infty}(0, t ; V) \cap L^{2}\left(0, t ; H^{3}(\Omega)\right)} \leq C_{20}\|u\|_{L^{2}(0, t ; H)} \text { for all } t \in[0, T] .
$$

Comparison in (2.21) together with an application of the mean value theorem and of (H5) then easily shows that also

$$
\|\mu\|_{L^{2}(0, t ; V)} \leq C_{21}\|u\|_{L^{2}(0, t ; H)} \quad \text { for all } t \in[0, T] .
$$

FOURTH ESTIMATE: Finally, we test (2.20) by an arbitrary function $v \in L^{2}(0, T ; V)$ to obtain that

$$
\begin{aligned}
& \left|\int_{0}^{t} \int_{\Omega} \partial_{t} \varphi v \mathrm{~d} x \mathrm{~d} s\right| \leq \int_{0}^{t} \int_{\Omega}|\nabla \mu||\nabla v| \mathrm{d} x \mathrm{~d} s+C_{22} \int_{0}^{t} \int_{\Omega}(|\sigma|+|\mu|)|v| \mathrm{d} x \mathrm{~d} s \\
& \quad+C_{23} \int_{0}^{t}\|\varphi(s)\|_{4}\left(\left\|\sigma_{2}(s)\right\|_{4}+\left\|\mu_{2}(s)\right\|_{4}\right)\|v(s)\|_{2} \mathrm{~d} x \mathrm{~d} s \\
& \leq C_{24}\left(\|\varphi\|_{L^{2}(0, t ; V)}+\|\mu\|_{L^{2}(0, t ; V)}+\|\sigma\|_{L^{2}(0, t ; H)}\right)\|v\|_{L^{2}(0, t ; V)} .
\end{aligned}
$$

In view of the above estimates, this implies that

$$
\left\|\partial_{t} \varphi\right\|_{L^{2}\left(0, t ; V^{\prime}\right)} \leq C_{25}\|u\|_{L^{2}(0, t ; H)} \quad \text { for all } t \in[0, T] .
$$

With (2.35), the assertion is completely proved. 


\section{Differentiability of the control-to-state operator}

In this section, we establish a differentiability result for the control-to-state operator $\mathcal{S}$. To this end, we assume that the general assumptions $(\mathbf{H 1})-(\mathbf{H 6})$ are satisfied. For arbitrary, but fixed $\bar{u} \in \mathcal{U}_{R}$, let $(\bar{\varphi}, \bar{\mu}, \bar{\sigma})=\mathcal{S}(\bar{u})$. We consider for any $h \in L^{2}(Q)$ the linearized system

$$
\begin{aligned}
& \partial_{t} \xi-\Delta \eta=P^{\prime}(\bar{\varphi})(\bar{\sigma}-\bar{\mu}) \xi+P(\bar{\varphi})(\rho-\eta) \text { in } Q, \\
& \eta=-\Delta \xi+F^{\prime \prime}(\bar{\varphi}) \xi \quad \text { in } Q, \\
& \partial_{t} \rho-\Delta \rho=-P^{\prime}(\bar{\varphi})(\bar{\sigma}-\bar{\mu}) \xi-P(\bar{\varphi})(\rho-\eta)+h \text { in } Q, \\
& \partial_{\mathbf{n}} \xi=\partial_{\mathbf{n}} \eta=\partial_{\mathbf{n}} \rho=0 \quad \text { on } \Sigma, \\
& \xi(0)=\rho(0)=0 \quad \text { in } \Omega .
\end{aligned}
$$

We expect the following: if the system (3.1) $-(3.5)$ admits a unique solution $(\xi, \eta, \rho)=$ : $\left(\xi^{h}, \eta^{h}, \rho^{h}\right)$ for every $h \in L^{2}(Q)$ and the Fréchet derivative $D \mathcal{S}(\bar{u})$ exists as a continuous linear mapping from $\mathcal{U}_{R}$ into a suitable Banach space (which is yet to be determined), then we should have $D \mathcal{S}(\bar{u}) h=\left(\xi^{h}, \eta^{h}, \rho^{h}\right)$. To this end, we first show the unique solvability of the linearized system (3.1)-(3.5). We have the following result.

THEOREM 3.1 The system (3.1)-(3.5) has a unique solution $(\xi, \eta, \rho)$ with

$$
\begin{aligned}
& \xi \in H^{1}\left(0, T ; V^{\prime}\right) \cap L^{\infty}(0, T ; V) \cap L^{2}\left(0, T ; W \cap H^{3}(\Omega)\right), \\
& \eta \in L^{2}(0, T ; V), \quad \rho \in H^{1}(0, T ; H) \cap C^{0}([0, T] ; V) \cap L^{2}(0, T ; W),
\end{aligned}
$$

which fulfills the conditions (3.1)-(3.5) almost everywhere in the respective sets, except for (3.1) and the related boundary condition in (3.4) that are fulfilled in the sense that, for almost every $t \in(0, T)$,

$$
\begin{aligned}
& \left\langle\partial_{t} \xi(t), v\right\rangle_{V}+\int_{\Omega} \nabla \eta(t) \cdot \nabla v \mathrm{~d} x=\int_{\Omega} P(\bar{\varphi}(t))(\rho(t)-\eta(t)) v \mathrm{~d} x \\
& +\int_{\Omega} P^{\prime}(\bar{\varphi}(t))(\bar{\sigma}(t)-\bar{\mu}(t)) \xi(t) v \mathrm{~d} x \quad \forall v \in V .
\end{aligned}
$$

Moreover, there is some constant $K_{4}^{*}>0$, which depends only on $R$ and the data of the state system, such that

$$
\begin{aligned}
& \|\xi\|_{H^{1}\left(0, t ; V^{\prime}\right) \cap L^{\infty}(0, t ; V) \cap L^{2}\left(0, t ; H^{3}(\Omega)\right)}+\|\eta\|_{L^{2}(0, t ; V)} \\
& +\|\rho\|_{H^{1}(0, t ; H) \cap C^{0}([0, t] ; V) \cap L^{2}\left(0, t ; H^{2}(\Omega)\right)} \leq K_{4}^{*}\|h\|_{L^{2}(0, t ; H)} \quad \text { for all } t \in[0, T] .
\end{aligned}
$$

REMARK 3 Note that an equivalent formulation of (3.7), which makes use of the abstract operator $A$ defined by (2.1), is

$$
\begin{aligned}
& \partial_{t} \xi(t)+A \eta(t)=P(\bar{\varphi}(t))(\rho(t)-\eta(t)) \\
& +P^{\prime}(\bar{\varphi}(t))(\bar{\sigma}(t)-\bar{\mu}(t)) \xi(t)+\eta(t) \quad \text { in } V^{\prime}, \text { for a.e. } t \in(0, T) .
\end{aligned}
$$


Proof. We apply a Faedo-Galerkin approximation, using the family $\left\{w_{j}\right\}_{j \in \mathbb{N}} \subset W$ of (appropriately ordered) eigenfunctions of the eigenvalue problem

$$
-\Delta w+w=\lambda w \quad \text { in } \Omega, \quad \partial_{\mathbf{n}} w=0 \quad \text { on } \partial \Omega,
$$

as a Galerkin basis in $V$. The family $\left\{w_{j}\right\}_{j \in \mathbb{N}}$ forms a complete orthonormal system in $\left(H,(\cdot, \cdot)_{H}\right)$ which is also orthogonal in $\left(V,(\cdot, \cdot)_{V}\right)$; moreover, we also have $\left(\Delta w_{j}, \Delta w_{k}\right)_{H}=0$ whenever $j \neq k$.

Let $n \in \mathbb{N}$ be fixed. Putting $\mathcal{W}_{n}:=\operatorname{span}\left\{w_{1}, \ldots, w_{n}\right\}$, we then look for functions of the form

$$
\xi_{n}(x, t):=\sum_{k=1}^{n} a_{k}^{n}(t) w_{k}(x), \quad \rho_{n}(x, t):=\sum_{k=1}^{n} b_{k}^{n}(t) w_{k}(x), \quad \eta_{n}(x, t):=\sum_{k=1}^{n} c_{k}^{n}(t) w_{k}(x),
$$

which satisfy the following approximating problem:

$$
\begin{aligned}
& \int_{\Omega} \partial_{t} \xi_{n}(t) v \mathrm{~d} x+\int_{\Omega} \nabla \eta_{n}(t) \cdot \nabla v \mathrm{~d} x-\int_{\Omega} P(\bar{\varphi}(t))\left(\rho_{n}(t)-\eta_{n}(t)\right) v \mathrm{~d} x \\
& =\int_{\Omega} P^{\prime}(\bar{\varphi}(t))(\bar{\sigma}(t)-\bar{\mu}(t)) \xi_{n}(t) v \mathrm{~d} x \quad \forall v \in \mathcal{W}_{n},
\end{aligned}
$$

$$
\begin{aligned}
& \int_{\Omega} \eta_{n}(t) v \mathrm{~d} x=\int_{\Omega} \nabla \xi_{n}(t) \cdot \nabla v \mathrm{~d} x+\int_{\Omega} F^{\prime \prime}(\bar{\varphi}(t)) \xi_{n}(t) v \mathrm{~d} x \quad \forall v \in \mathcal{W}_{n}, \\
& \int_{\Omega} \partial_{t} \rho_{n}(t) v \mathrm{~d} x+\int_{\Omega} \nabla \rho_{n}(t) \cdot \nabla v \mathrm{~d} x+\int_{\Omega} P(\bar{\varphi}(t))\left(\rho_{n}(t)-\eta_{n}(t)\right) v \mathrm{~d} x \\
& =\int_{\Omega}\left[-P^{\prime}(\bar{\varphi}(t))(\bar{\sigma}(t)-\bar{\mu}(t)) \xi_{n}(t)+h(t)\right] v \mathrm{~d} x \quad \forall v \in \mathcal{W}_{n}, \\
& \xi_{n}(0)=\rho_{n}(0)=0 .
\end{aligned}
$$

By insertion of $v=w_{j}, 1 \leq j \leq n$, in (3.11), it is easily seen that the unknowns $c_{j}^{n}$ can be expressed explicitly in terms of the unknowns $a_{1}^{n}, \ldots, a_{n}^{n}$; hence, by inserting $v=w_{j}, 1 \leq j \leq n$, in (3.10) and (3.12), it turns out that the system (3.10)-(3.13) is in fact equivalent to a Cauchy problem for a linear system of $2 n$ first-order ordinary differential equations in the $2 n$ unknowns $a_{j}^{n}, b_{j}^{n}, 1 \leq j \leq n$, in which, owing to $(\mathbf{H} 4)$ and (H5), and to the fact that $\bar{\varphi} \in C^{0}(\bar{Q})$ (recall (2.17)), all of the occurring coefficient functions belong to $L^{\infty}(0, T)$. By Carathéodory's theorem, this linear system has a unique solution $\left(a_{1}^{n}, \ldots, a_{n}^{n}, b_{1}^{n}, \ldots, b_{n}^{n}\right) \in\left(W^{1, \infty}(0, T)\right)^{2 n}$, which specifies the unique solution $\left(\xi_{n}, \eta_{n}, \rho_{n}\right) \in\left(W^{1, \infty}\left(0, T ; \mathcal{W}_{n}\right)\right)^{3}$ to (3.10)-(3.13).

We now aim to derive a number of a priori estimates for the approximations. To this end, we denote by $C_{i}, i \in \mathbb{N}$, positive constants (possibly different from the ones used in the previous section) that may depend on the data of the problem, but not on $n \in \mathbb{N}$. 
FIRST ESTIMATE: Let $t \in[0, T]$ be arbitrary. We insert $v=\eta_{n}$ in (3.10), $v=-\partial_{t} \xi_{n}$ in (3.11), as well as $v=\rho_{n}$ in (3.12), and add the resulting identities. Integrating over $\Omega \times[0, t]$ and by parts, we obtain that

$$
\begin{aligned}
& \frac{1}{2}\left(\left\|\rho_{n}(t)\right\|_{H}^{2}+\left\|\nabla \xi_{n}(t)\right\|_{H}^{2}\right)+\int_{0}^{t} \int_{\Omega}\left(\left|\nabla \rho_{n}\right|^{2}+\left|\nabla \eta_{n}\right|^{2}\right) \mathrm{d} x \mathrm{~d} s \\
+ & \int_{0}^{t} \int_{\Omega} P(\bar{\varphi})\left(\rho_{n}-\eta_{n}\right)^{2} \mathrm{~d} x \mathrm{~d} s=-\int_{0}^{t} \int_{\Omega} \partial_{t} \xi_{n} F^{\prime \prime}(\bar{\varphi}) \xi_{n} \mathrm{~d} x \mathrm{~d} s \\
+ & \int_{0}^{t} \int_{\Omega} h \rho_{n} \mathrm{~d} x \mathrm{~d} s+\int_{0}^{t} \int_{\Omega} P^{\prime}(\bar{\varphi})(\bar{\sigma}-\bar{\mu}) \xi_{n}\left(\eta_{n}-\rho_{n}\right) \mathrm{d} x \mathrm{~d} s .
\end{aligned}
$$

Owing to $(\mathbf{H} 4)$, the last integral on the left is nonnegative. We denote the integrals on the right by $I_{i}, i=1,2,3$, in that order, and estimate them indivually. Clearly, by Young's inequality, it turns out that

$$
\left|I_{2}\right| \leq \frac{1}{2}\left(\|h\|_{L^{2}(0, t ; H)}^{2}+\left\|\rho_{n}\right\|_{L^{2}(0, t ; H)}^{2}\right) .
$$

Moreover, by virtue of (2.17) and Hölder's and Young's inequalities, we have that

$$
\begin{aligned}
& \left|I_{1}\right|=\left.\left|-\frac{1}{2} \int_{\Omega} F^{\prime \prime}(\bar{\varphi}(t))\right| \xi_{n}(t)\right|^{2} \mathrm{~d} x+\frac{1}{2} \int_{0}^{t} \int_{\Omega} F^{\prime \prime \prime}(\bar{\varphi}) \partial_{t} \bar{\varphi} \xi_{n}^{2} \mathrm{~d} x \mathrm{~d} s \mid \\
& \leq \frac{K_{1}^{*}}{2}\left\|\xi_{n}(t)\right\|_{H}^{2}+C_{1} \int_{0}^{t}\left\|\partial_{t} \bar{\varphi}(s)\right\|_{6}\left\|\xi_{n}(s)\right\|_{3}\left\|\xi_{n}(s)\right\|_{2} \mathrm{~d} s \\
& \leq \frac{K_{1}^{*}}{2}\left\|\xi_{n}(t)\right\|_{H}^{2}+C_{2} \int_{0}^{t}\left(\left\|\xi_{n}(s)\right\|_{V}^{2}+\left\|\partial_{t} \bar{\varphi}(s)\right\|_{V}^{2}\left\|\xi_{n}(s)\right\|_{H}^{2}\right) \mathrm{d} s,
\end{aligned}
$$

where we notice that the mapping $s \mapsto\left\|\partial_{t} \bar{\varphi}(s)\right\|_{V}^{2}$ belongs to $L^{1}(0, T)$, due to (2.9)). Next, we observe that, for every $\gamma>0$ (to be chosen later),

$$
\begin{aligned}
\left|I_{3}\right| & \leq C_{3} \int_{0}^{t}\left(\|\bar{\sigma}(s)\|_{6}+\|\bar{\mu}(s)\|_{6}\right)\left\|\xi_{n}(s)\right\|_{3}\left(\left\|\eta_{n}(s)\right\|_{2}+\left\|\rho_{n}(s)\right\|_{2}\right) \mathrm{d} s \\
& \leq \gamma\left(\left\|\eta_{n}\right\|_{L^{2}(0, t ; H)}^{2}+\left\|\rho_{n}\right\|_{L^{2}(0, t ; H)}^{2}\right)+\frac{C_{4}}{\gamma}\left\|\xi_{n}(s)\right\|_{L^{2}(0, t ; V)}^{2} .
\end{aligned}
$$

We still need estimates for the $L^{2}(Q)$ norm of $\eta_{n}$ and for the $L^{\infty}(0, T ; H)$ norm of $\xi_{n}$. To obtain the former, we insert $v=\eta_{n}$ in (3.11), integrate over $[0, t]$ and by parts, and apply Young's inequality and (2.17) to deduce that

$$
\begin{aligned}
& \int_{0}^{t} \int_{\Omega} \eta_{n}^{2} \mathrm{~d} x \mathrm{~d} s=\int_{0}^{t} \int_{\Omega} \eta_{n}\left(-\Delta \xi_{n}+F^{\prime \prime}(\bar{\varphi}) \xi_{n}\right) \mathrm{d} x \mathrm{~d} s \\
& \leq \frac{1}{2} \int_{0}^{t} \int_{\Omega} \eta_{n}^{2} \mathrm{~d} x \mathrm{~d} s+\frac{1}{2} \int_{0}^{t} \int_{\Omega}\left(\left|\Delta \xi_{n}\right|+K_{1}^{*}\left|\xi_{n}\right|\right)^{2} \mathrm{~d} x \mathrm{~d} s
\end{aligned}
$$

whence

$$
\left\|\eta_{n}\right\|_{L^{2}(0, t ; H)}^{2} \leq 2 \int_{0}^{t} \int_{\Omega}\left|\Delta \xi_{n}\right|^{2} \mathrm{~d} x \mathrm{~d} s+C_{5}\left\|\xi_{n}\right\|_{L^{2}(0, t ; H)}^{2}
$$


To derive the missing estimates, we finally insert $v=\left(2+K_{1}^{*}\right) \xi_{n}$ in (3.10) and $v=$ $\left(2+K_{1}^{*}\right) \Delta \xi_{n}$ in (3.11) and add the resulting equations. Integration over $[0, t]$ and by parts then yields that

$$
\begin{aligned}
& \left(1+\frac{K_{1}^{*}}{2}\right)\left\|\xi_{n}(t)\right\|_{H}^{2}+\left(2+K_{1}^{*}\right) \int_{0}^{t} \int_{\Omega}\left|\Delta \xi_{n}\right|^{2} \mathrm{~d} x \mathrm{~d} s \\
& =\int_{0}^{t} \int_{\Omega}\left(2+K_{1}^{*}\right) P(\bar{\varphi})\left(\rho_{n}-\eta_{n}\right) \xi_{n} \mathrm{~d} x \mathrm{~d} s \\
& \quad+\int_{0}^{t} \int_{\Omega}\left(2+K_{1}^{*}\right) P^{\prime}(\bar{\varphi})(\bar{\sigma}-\bar{\mu}) \xi_{n}^{2} \mathrm{~d} x \mathrm{~d} s \\
& \quad+\int_{0}^{t} \int_{\Omega}\left(2+K_{1}^{*}\right) F^{\prime \prime}(\bar{\varphi}) \xi_{n} \Delta \xi_{n} \mathrm{~d} x \mathrm{~d} s .
\end{aligned}
$$

We denote the integrals on the right-hand side by $I_{4}, I_{5}, I_{6}$, in this order, and estimate them individually. First, we obviously have

$$
\left|I_{6}\right| \leq \frac{K_{1}^{*}}{2} \int_{0}^{t} \int_{\Omega}\left|\Delta \xi_{n}\right|^{2} \mathrm{~d} x \mathrm{~d} s+C_{6} \int_{0}^{t} \int_{\Omega}\left|\xi_{n}\right|^{2} \mathrm{~d} x \mathrm{~d} s .
$$

Moreover, owing to Hölder's and Young's inequalities and (2.17), we infer that

$$
\left|I_{5}\right| \leq C_{7} \int_{0}^{t}\left(\|\bar{\sigma}(s)\|_{6}+\|\bar{\mu}(s)\|_{6}\right)\left\|\xi_{n}(s)\right\|_{3}\left\|\xi_{n}(s)\right\|_{2} \mathrm{~d} s \leq C_{8}\left\|\xi_{n}\right\|_{L^{2}(0, t ; V)}^{2},
$$

and, using Young's inequality once more,

$$
\left|I_{4}\right| \leq \gamma\left(\left\|\eta_{n}\right\|_{L^{2}(0, t ; H)}^{2}+\left\|\rho_{n}\right\|_{L^{2}(0, t ; H)}^{2}\right)+\frac{C_{9}}{\gamma}\left\|\xi_{n}\right\|_{L^{2}(0, t ; H)}^{2} .
$$

Now, we take the sum of (3.14), (3.18), and (3.19). Then, on account of (3.15) $-(3.17)$ and of (3.20)-(3.22), we can choose $\gamma$ small enough (namely, $\gamma<1 / 2$ ) and apply Gronwall's lemma in order to find the estimate

$$
\begin{aligned}
& \left\|\xi_{n}\right\|_{L^{\infty}(0, t ; V) \cap L^{2}\left(0, t ; H^{2}(\Omega)\right)}+\left\|\eta_{n}\right\|_{L^{2}(0, t ; V)}+\left\|\rho_{n}\right\|_{L^{\infty}(0, t ; H) \cap L^{2}(0, t ; V)} \\
& \leq C_{10}\|h\|_{L^{2}(0, t ; H)} \text { for all } t \in[0, T] .
\end{aligned}
$$

SeCond Estimate: Let $t \in[0, T]$ be arbitrary. Observing that $v=\Delta^{2} \xi_{n} \in \mathcal{W}_{n}$, we obtain from (3.11), using integration by parts, Young's inequality, and (2.17), that

$$
\begin{aligned}
& \int_{0}^{t} \int_{\Omega}\left|\nabla \Delta \xi_{n}\right|^{2} \mathrm{~d} x \mathrm{~d} s=\int_{0}^{t} \int_{\Omega} \nabla \xi_{n} \cdot \nabla \Delta^{2} \xi_{n} \mathrm{~d} x \mathrm{~d} s=\int_{0}^{t} \int_{\Omega} \Delta^{2} \xi_{n}\left(\eta_{n}-F^{\prime \prime}(\bar{\varphi}) \xi_{n}\right) \mathrm{d} x \mathrm{~d} s \\
& =-\int_{0}^{t} \int_{\Omega} \nabla \Delta \xi_{n} \cdot\left(\nabla \eta_{n}-\xi_{n} F^{\prime \prime \prime}(\bar{\varphi}) \nabla \bar{\varphi}-F^{\prime \prime}(\bar{\varphi}) \nabla \xi_{n}\right) \mathrm{d} x \mathrm{~d} s \\
& \leq \frac{1}{2} \int_{0}^{t} \int_{\Omega}\left|\nabla \Delta \xi_{n}\right|^{2} \mathrm{~d} x \mathrm{~d} s+C_{11} \int_{0}^{t} \int_{\Omega}\left(\left|\nabla \eta_{n}\right|^{2}+\left|\xi_{n}\right|^{2}+\left|\nabla \xi_{n}\right|^{2}\right) \mathrm{d} x \mathrm{~d} s,
\end{aligned}
$$


and it follows from (3.23) that

$$
\left\|\xi_{n}\right\|_{L^{2}\left(0, t ; H^{3}(\Omega)\right)} \leq C_{12}\|h\|_{L^{2}(0, t ; H)} \quad \text { for all } t \in[0, T] .
$$

ThiRd estimate: Let $t \in[0, T]$ be arbitrary. We insert $v=\partial_{t} \rho_{n}$ in (3.12) and integrate over $[0, t]$ and by parts. Using (2.17) and Young's and Hölder's inequalities, we obtain for every $\gamma>0$ the estimate

$$
\begin{aligned}
& \int_{0}^{t} \int_{\Omega}\left|\partial_{t} \rho_{n}\right|^{2} \mathrm{~d} x \mathrm{~d} s+\frac{1}{2}\left\|\nabla \rho_{n}(t)\right\|_{H}^{2} \leq \gamma \int_{0}^{t} \int_{\Omega}\left|\partial_{t} \rho_{n}\right|^{2} \mathrm{~d} x \mathrm{~d} s \\
& +\frac{C_{13}}{\gamma} \int_{0}^{t} \int_{\Omega}\left(\rho_{n}^{2}+\eta_{n}^{2}+h^{2}\right) \mathrm{d} x \mathrm{~d} s+C_{14} I
\end{aligned}
$$

holds, where

$$
\begin{aligned}
I & :=\int_{0}^{t}\left(\|\bar{\sigma}(s)\|_{6}+\|\bar{\mu}(s)\|_{6}\right)\left\|\xi_{n}(s)\right\|_{3}\left\|\partial_{t} \rho_{n}(s)\right\|_{2} \mathrm{~d} s \\
& \leq \gamma \int_{0}^{t} \int_{\Omega}\left|\partial_{t} \rho_{n}\right|^{2} \mathrm{~d} x \mathrm{~d} s+\frac{C_{15}}{\gamma}\left\|\xi_{n}\right\|_{L^{2}(0, t ; V)}^{2},
\end{aligned}
$$

Choosing $1 / 2>\gamma>0$, we obtain from (3.23) that

$$
\left\|\rho_{n}\right\|_{H^{1}(0, t ; H) \cap L^{\infty}(0, t ; V)} \leq C_{16}\|h\|_{L^{2}(0, t ; H)} \quad \text { for all } t \in[0, T] .
$$

Similar reasoning, using $v=-\Delta \rho_{n}$ in (3.12), yields that also

$$
\int_{0}^{t} \int_{\Omega}\left|\Delta \rho_{n}\right|^{2} \mathrm{~d} x \mathrm{~d} s \leq C_{17}\|h\|_{L^{2}(0, t ; H)}^{2}
$$

so that

$$
\left\|\rho_{n}\right\|_{L^{2}\left(0, t ; H^{2}(\Omega)\right)} \leq C_{18}\|h\|_{L^{2}(0, t ; H)} .
$$

In conclusion, we have shown the estimate

$$
\begin{aligned}
& \left\|\xi_{n}\right\|_{L^{\infty}(0, t ; V) \cap L^{2}\left(0, t ; H^{3}(\Omega)\right)}+\left\|\eta_{n}\right\|_{L^{2}(0, t ; V)} \\
& +\left\|\rho_{n}\right\|_{H^{1}(0, t ; H) \cap C^{0}([0, t] ; V) \cap L^{2}\left(0, t ; H^{2}(\Omega)\right)} \leq C_{19}\|h\|_{L^{2}(0, t ; H)} .
\end{aligned}
$$

CONCLUSION OF THE PROOF: It follows from (3.27) that there are functions $(\xi, \eta, \rho)$ such that, possibly only for a subsequence which is again indexed by $n$,

$$
\begin{array}{ll}
\xi_{n} \rightarrow \xi & \text { weakly in } L^{2}\left(0, T ; H^{3}(\Omega)\right) \\
& \text { and weakly star in } L^{\infty}(0, T ; V), \\
\eta_{n} \rightarrow \eta & \text { weakly in } L^{2}(0, T ; V), \\
\rho_{n} \rightarrow \rho & \text { weakly in } H^{1}(0, T ; H) \cap L^{2}\left(0, T ; H^{2}(\Omega)\right) .
\end{array}
$$


From the semicontinuity properties of the involved norms, we can infer that the estimate (3.27) holds true for $(\xi, \eta, \rho)$ in place of $\left(\xi_{n}, \eta_{n}, \rho_{n}\right)$, and it is easily seen that $(\xi, \eta, \rho)$ satisfies (3.2) and (3.3) almost everywhere in $Q$. Moreover, we have $\rho(0)=0$ almost everywhere in $\Omega$ and $\partial_{\mathbf{n}} \xi=\partial_{\mathbf{n}} \rho=0$ almost everywhere on $\Sigma$, and it follows that, for every $v \in H^{1}(0, T ; V)$ with $v(T)=0$, it holds the identity

$$
\begin{aligned}
& -\int_{0}^{T} \int_{\Omega} \xi \partial_{t} v \mathrm{~d} x \mathrm{~d} t+\int_{0}^{T} \int_{\Omega} \nabla \eta \cdot \nabla v \mathrm{~d} x \mathrm{~d} t \\
& =\int_{0}^{T} \int_{\Omega}\left(P^{\prime}(\bar{\varphi})(\bar{\sigma}-\bar{\mu}) \xi+P(\bar{\varphi})(\rho-\eta)\right) v \mathrm{~d} x \mathrm{~d} t,
\end{aligned}
$$

which implies that (3.7) and $\xi(0)=0$ hold true. Indeed, we also recover that $\xi \in$ $H^{1}\left(0, T ; V^{\prime}\right)$, and in addition comparison yields

$$
\|\xi\|_{H^{1}\left(0, t ; V^{\prime}\right)} \leq C_{20}\|h\|_{L^{2}(0, t ; H)},
$$

so that (3.8) is shown.

To prove uniqueness, we write the system (3.7), (3.2)-(3.5) for two solutions $\left(\xi_{i}, \eta_{i}, \rho_{i}\right), i=1,2$, and subtract the equations. Then $\xi:=\xi_{1}-\xi_{2}, \eta:=\eta_{1}-\eta_{2}$, $\rho:=\rho_{1}-\rho_{2}$ satisfy the system (3.7), (3.2)-(3.5) with $h \equiv 0$. Now notice that, up to obvious modifications which are necessary due to the fact that we only have $\partial_{t} \xi \in L^{2}\left(0, T ; V^{\prime}\right)$, the estimates leading to (3.23) can be repeated. We point out, in particular, that all the three terms of the equation (3.2) belong to $L^{2}(0, T ; V)$ thanks to (3.6)), (H5) and (2.8). Then, since $h \equiv 0$ in this case, we must have $\xi=\eta=\rho=0$ and thus uniqueness.

We are now in a position to establish the Fréchet differentiability of the controlto-state operator. We have the following result.

ThEOREM $3.2 \quad$ Suppose that the assumptions $(\mathbf{H 1})-(\mathbf{H 6})$ are satisfied. Then the control-to-state mapping $\mathcal{S}$ is Fréchet differentiable in $\mathcal{U}_{R}$ as a mapping from $L^{2}(Q)$ into the space

$$
\begin{aligned}
\mathcal{Y}:=( & \left.H^{1}\left(0, T ; W^{\prime}\right) \cap L^{\infty}(0, T ; H) \cap L^{2}(0, T ; W)\right) \times L^{2}(Q) \\
& \times\left(H^{1}(0, T ; H) \cap L^{2}\left(0, T ; H^{2}(\Omega)\right)\right) .
\end{aligned}
$$

Moreover, for any $\bar{u} \in \mathcal{U}_{R}$ the Fréchet derivative $D \mathcal{S}(\bar{u}) \in \mathcal{L}\left(L^{2}(Q), \mathcal{Y}\right)$ is defined as follows: for any $h \in L^{2}(Q)$, we have $D \mathcal{S}(\bar{u}) h=\left(\xi^{h}, \eta^{h}, \rho^{h}\right)$, where $\left(\xi^{h}, \eta^{h}, \rho^{h}\right)$ is the unique solution to the linearized system (3.1)-(3.5) associated with $h$.

Proof: Let $\bar{u} \in \mathcal{U}_{R}$ be arbitrary, and $(\bar{\varphi}, \bar{\mu}, \bar{\sigma})=\mathcal{S}(\bar{u})$. Since $\mathcal{U}_{R}$ is open, there is some $\Lambda>0$ such that $\bar{u}+h \in \mathcal{U}_{R}$ whenever $h \in L^{2}(Q)$ and $\|h\|_{L^{2}(Q)} \leq \Lambda$. In the following, we only consider such variations $h \in L^{2}(Q)$. We put $\left(\varphi^{h}, \mu^{h}, \sigma^{h}\right):=$ $\mathcal{S}(\bar{u}+h)$, and we denote by $\left(\xi^{h}, \eta^{h}, \rho^{h}\right)$ the unique solution to the linearized system (3.1) - (3.5) associated with $h$. Notice that by (3.8) the linear mapping $h \mapsto\left(\xi^{h}, \eta^{h}, \rho^{h}\right)$ is continuous between the spaces $L^{2}(Q)$ and $\mathcal{Y}$. We now define

$$
\psi^{h}:=\varphi^{h}-\bar{\varphi}-\xi^{h}, \quad \zeta^{h}:=\mu^{h}-\bar{\mu}-\eta^{h}, \quad \chi^{h}:=\sigma^{h}-\bar{\sigma}-\rho^{h} .
$$


According to Theorem 2.1 and Theorem 3.1, we have the regularity

$$
\begin{aligned}
& \psi^{h} \in H^{1}\left(0, T ; V^{\prime}\right) \cap L^{\infty}(0, T ; V) \cap L^{2}\left(0, T ; H^{3}(\Omega)\right), \quad \zeta^{h} \in L^{2}(0, T ; V), \\
& \chi^{h} \in H^{1}(0, T ; H) \cap C^{0}([0, T] ; V) \cap L^{2}\left(0, T ; H^{2}(\Omega)\right) .
\end{aligned}
$$

Note also that $\left(\varphi^{h}, \mu^{h}, \sigma^{h}\right)$ and $(\bar{\varphi}, \bar{\mu}, \bar{\sigma})$ satisfy the global bounds (2.9) and (2.17). Let us point out that $\psi^{h}$ is at least strongly continuous from $[0, T]$ to $H$ (see, e.g., [39, Sec. 8, Cor. 4]).

According to the definition of Fréchet differentiability, it suffices to show that there exists an increasing function $Z:(0,+\infty) \rightarrow(0,+\infty)$ with $\lim _{\lambda \searrow 0} Z(\lambda) / \lambda^{2}=0$ and

$$
\left\|\left(\psi^{h}, \zeta^{h}, \chi^{h}\right)\right\|_{\mathcal{Y}}^{2} \leq Z\left(\|h\|_{L^{2}(Q)}\right)
$$

Now observe that $\left(\psi^{h}, \zeta^{h}, \chi^{h}\right)$ is a solution to the following problem:

$$
\begin{gathered}
\left\langle\partial_{t} \psi^{h}(t), v\right\rangle_{V}+\int_{\Omega} \nabla \zeta^{h}(t) \cdot \nabla v \mathrm{~d} x=\int_{\Omega}\left[P\left(\varphi^{h}\right)\left(\sigma^{h}-\mu^{h}\right)-P(\bar{\varphi})(\bar{\sigma}-\bar{\mu})\right. \\
\left.-P^{\prime}(\bar{\varphi})(\bar{\sigma}-\bar{\mu}) \xi^{h}-P(\bar{\varphi})\left(\rho^{h}-\eta^{h}\right)\right](t) v \mathrm{~d} x \quad \text { for all } v \in V \text { and a. e. } t \in(0, T), \\
\zeta^{h}=-\Delta \psi^{h}+F^{\prime}\left(\varphi^{h}\right)-F^{\prime}(\bar{\varphi})-F^{\prime \prime}(\bar{\varphi}) \xi^{h} \quad \text { a. e in } Q, \\
\partial_{t} \chi^{h}-\Delta \chi^{h}=-P\left(\varphi^{h}\right)\left(\sigma^{h}-\mu^{h}\right)+P(\bar{\varphi})(\bar{\sigma}-\bar{\mu})+P^{\prime}(\bar{\varphi})(\bar{\sigma}-\bar{\mu}) \xi^{h} \\
+P(\bar{\varphi})\left(\rho^{h}-\eta^{h}\right) \quad \text { a.e. in } Q,
\end{gathered}
$$

$$
\begin{aligned}
& \partial_{\mathbf{n}} \psi^{h}=\partial_{\mathbf{n}} \chi^{h}=0 \quad \text { a.e. on } \Sigma, \\
& \psi^{h}(0)=\chi^{h}(0)=0 \quad \text { a.e. in } \Omega .
\end{aligned}
$$

We also note that a straightforward computation, using Taylor's theorem with integral remainder, yields the identities

$$
\begin{aligned}
& F^{\prime}\left(\varphi^{h}\right)-F^{\prime}(\bar{\varphi})-F^{\prime \prime}(\bar{\varphi}) \xi^{h}=F^{\prime \prime}(\bar{\varphi}) \psi^{h}+R_{1}^{h}\left(\varphi^{h}-\bar{\varphi}\right)^{2}, \\
& P\left(\varphi^{h}\right)\left(\sigma^{h}-\mu^{h}\right)-P(\bar{\varphi})(\bar{\sigma}-\bar{\mu})-P^{\prime}(\bar{\varphi})(\bar{\sigma}-\bar{\mu}) \xi^{h}-P(\bar{\varphi})\left(\rho^{h}-\eta^{h}\right) \\
&=P\left(\varphi^{h}\right) \sigma^{h}-P\left(\varphi^{h}\right) \mu^{h}-P(\bar{\varphi}) \bar{\sigma}+P(\bar{\varphi}) \bar{\mu}-P^{\prime}(\bar{\varphi}) \bar{\sigma} \xi^{h}+P^{\prime}(\bar{\varphi}) \bar{\mu} \xi^{h} \\
& \quad-P(\bar{\varphi})\left(\sigma^{h}-\bar{\sigma}-\chi^{h}\right)+P(\bar{\varphi})\left(\mu^{h}-\bar{\mu}-\zeta^{h}\right) \\
&=P(\bar{\varphi}) \chi^{h}+\left(P\left(\varphi^{h}\right)-P(\bar{\varphi})\right)\left(\sigma^{h}-\bar{\sigma}\right)+\left(P\left(\varphi^{h}\right)-P(\bar{\varphi})-P^{\prime}(\bar{\varphi}) \xi^{h}\right) \bar{\sigma} \\
& \quad-P(\bar{\varphi}) \zeta^{h}-\left(P\left(\varphi^{h}\right)-P(\bar{\varphi})\right)\left(\mu^{h}-\bar{\mu}\right)-\left(P\left(\varphi^{h}\right)-P(\bar{\varphi})-P^{\prime}(\bar{\varphi}) \xi^{h}\right) \bar{\mu} \\
&=P(\bar{\varphi})\left(\chi^{h}-\zeta^{h}\right)+\left(P\left(\varphi^{h}\right)-P(\bar{\varphi})\right)\left[\left(\sigma^{h}-\bar{\sigma}\right)-\left(\mu^{h}-\bar{\mu}\right)\right] \\
& \quad+P^{\prime}(\bar{\varphi})(\bar{\sigma}-\bar{\mu}) \psi^{h}+(\bar{\sigma}-\bar{\mu}) R_{2}^{h}\left(\varphi^{h}-\bar{\varphi}\right)^{2}=: Q^{h},
\end{aligned}
$$

where

$$
R_{1}^{h}=\int_{0}^{1}(1-z) F^{\prime \prime \prime}\left(\bar{\varphi}+z\left(\varphi^{h}-\bar{\varphi}\right)\right) \mathrm{d} z, \quad R_{2}^{h}=\int_{0}^{1}(1-z) P^{\prime \prime}\left(\bar{\varphi}+z\left(\varphi^{h}-\bar{\varphi}\right)\right) \mathrm{d} z
$$


In the following estimates, we denote by $C_{i}, i \in \mathbb{N}$, positive constants (possibly different from the ones used in the previous sections) which may depend on the data of the state system but not on $h \in L^{2}(Q)$ with $\|h\|_{L^{2}(Q)} \leq \Lambda$. For the sake of a better readability, we will often suppress the superscript $h$ in the functions $\left(\psi^{h}, \zeta^{h}, \chi^{h}\right)$ during the estimates and only write them in the final estimate in each step. We first notice that, thanks to (H4), (H5), and (2.17),

$$
\left\|R_{1}^{h}\right\|_{L^{\infty}(Q)}+\left\|R_{2}^{h}\right\|_{L^{\infty}(Q)} \leq C_{1} .
$$

We also recall that the inequalities (1.9)-(1.13), the global bounds (2.9), (2.17), the global stability estimates (2.10), (2.18), (2.19), and the properties (2.2), (2.3) satisfied by the Riesz isomorphism $A$ introduced in (2.1) will be frequently used in the sequel without mentioning them. We begin our analysis by proving some preparatory $L^{2}$ estimates. We have, for every $t \in[0, T]$ :

$$
\begin{aligned}
& \int_{0}^{t}\left\|R_{1}^{h}(s)\left(\varphi^{h}(s)-\bar{\varphi}(s)\right)^{2}\right\|_{H}^{2} \mathrm{~d} s \leq C_{2} \int_{0}^{t} \int_{\Omega}\left|\varphi^{h}-\bar{\varphi}\right|^{4} \mathrm{~d} x \mathrm{~d} s \\
& \leq C_{2} \int_{0}^{t}\left\|\varphi^{h}(s)-\bar{\varphi}(s)\right\|_{\infty}^{2}\left\|\varphi^{h}(s)-\bar{\varphi}(s)\right\|_{H}^{2} \mathrm{~d} s \leq C_{3}\|h\|_{L^{2}(0, t ; H)}^{4} .
\end{aligned}
$$

Moreover, owing to (1.10), (2.9), (2.17), (H4), and (2.19), we infer that

$$
\begin{aligned}
& \int_{0}^{t}\left\|\left(P\left(\varphi^{h}(s)\right)-P(\bar{\varphi}(s))\right)\left(\left(\sigma^{h}(s)-\bar{\sigma}(s)\right)-\left(\mu^{h}(s)-\bar{\mu}(s)\right)\right)\right\|_{H}^{2} \mathrm{~d} s \\
& \leq C_{4} \int_{0}^{t}\left\|P\left(\varphi^{h}(s)\right)-P(\bar{\varphi}(s))\right\|_{V}^{2}\left(\left\|\sigma^{h}(s)-\bar{\sigma}(s)\right\|_{V}^{2}+\left\|\mu^{h}(s)-\bar{\mu}(s)\right\|_{V}^{2}\right) \mathrm{d} s \\
& \leq C_{5} \int_{0}^{t}\left\|\varphi^{h}(s)-\bar{\varphi}(s)\right\|_{V}^{2}\left(\left\|\sigma^{h}(s)-\bar{\sigma}(s)\right\|_{V}^{2}+\left\|\mu^{h}(s)-\bar{\mu}(s)\right\|_{V}^{2}\right) \mathrm{d} s \\
& \leq C_{6}\|h\|_{L^{2}(0, t ; H)}^{4},
\end{aligned}
$$

as well as, using Hölder's inequality, (2.17), and (2.19),

$$
\begin{aligned}
& \int_{0}^{t}\left\|(\bar{\sigma}(s)-\bar{\mu}(s)) R_{2}^{h}(s)\left(\varphi^{h}(s)-\bar{\varphi}(s)\right)^{2}\right\|_{H}^{2} \mathrm{~d} s \\
& \leq C_{7} \int_{0}^{t} \int_{\Omega}\left(|\bar{\sigma}|^{2}+|\bar{\mu}|^{2}\right)\left|\varphi^{h}-\bar{\varphi}\right|^{4} \mathrm{~d} x \mathrm{~d} s \\
& \leq C_{8} \int_{0}^{t}\left(\|\bar{\sigma}(s)\|_{6}^{2}+\|\bar{\mu}(s)\|_{6}^{2}\right)\left\|\varphi^{h}(s)-\bar{\varphi}(s)\right\|_{6}^{4} \mathrm{~d} s \leq C_{9}\|h\|_{L^{2}(0, t ; H)}^{4} .
\end{aligned}
$$

Moreover, we have that

$$
\begin{aligned}
& \int_{0}^{t}\left\|P^{\prime}(\bar{\varphi}(s))(\bar{\sigma}(s)-\bar{\mu}(s)) \psi(s)\right\|_{H}^{2} \mathrm{~d} s \leq C_{10} \int_{0}^{t} \int_{\Omega}\left(|\bar{\sigma}|^{2}+|\bar{\mu}|^{2}\right)|\psi|^{2} \mathrm{~d} x \mathrm{~d} s \\
& \leq C_{11} \int_{0}^{t}\left(\|\bar{\sigma}(s)\|_{6}^{2}+\|\bar{\mu}(s)\|_{6}^{2}\right)\|\psi(s)\|_{3}^{2} \mathrm{~d} s \leq C_{12} \int_{0}^{t}\|\psi(s)\|_{V}^{2} \mathrm{~d} s .
\end{aligned}
$$


First estimate: First, we observe that Eqs. (3.31)-(3.33) can be rewritten in the form

$$
\begin{aligned}
& \partial_{t} \psi+A \zeta=Q^{h}+\zeta, \quad \zeta=A \psi+F^{\prime \prime}(\bar{\varphi}) \psi+R_{1}^{h}\left(\varphi^{h}-\bar{\varphi}\right)^{2}-\psi, \\
& \partial_{t} \chi+A \chi=-Q^{h}+\chi \quad \text { in } V^{\prime}, \quad \text { a.e. in }(0, T),
\end{aligned}
$$

where $Q^{h}$ is defined in (3.37).

We now test the first equation in (3.44) by $A^{-1} \psi$, the third by $A^{-1} \chi$ and add the resulting identities. Using (2.2) and (2.3), we easily deduce that, for any $t \in[0, T]$,

$$
\begin{aligned}
& \frac{1}{2}\left(\|\psi(t)\|_{V^{\prime}}^{2}+\|\chi(t)\|_{V^{\prime}}^{2}\right)+\int_{0}^{t} \int_{\Omega}\left(|\nabla \psi|^{2}+|\chi|^{2}\right) \mathrm{d} x \mathrm{~d} s \\
& \leq-\int_{0}^{t} \int_{\Omega} F^{\prime \prime}(\bar{\varphi}) \psi^{2} \mathrm{~d} x \mathrm{~d} s-\int_{0}^{t} \int_{\Omega} R_{1}^{h}\left(\varphi^{h}-\bar{\varphi}\right)^{2} \psi \mathrm{d} x \mathrm{~d} s+\int_{0}^{t}\|\chi(s)\|_{V^{\prime}}^{2} \mathrm{~d} s \\
& \quad+\int_{0}^{t}\left\langle Q^{h}(s), A^{-1} \psi(s)-A^{-1} \chi(s)\right\rangle_{V} \mathrm{~d} s+\int_{0}^{t}\left\langle\zeta(s), A^{-1} \psi(s)\right\rangle_{V} \mathrm{~d} s
\end{aligned}
$$

We denote the first, second, fourth, and fifth integral on the right-hand side by $I_{1}, I_{2}, I_{3}, I_{4}$, in this order. Using (2.17), (3.40), and Young's inequality, we have

$$
\left|I_{1}\right|+\left|I_{2}\right| \leq C_{13}\left(\|\psi\|_{L^{2}(0, t ; H)}^{2}+\|h\|_{L^{2}(0, t ; H)}^{4}\right) \text {. }
$$

Using (3.44) and (3.40), we also have

$$
\begin{aligned}
\left|I_{4}\right| & =\left|\int_{0}^{t}\left\langle\left(A \psi-\psi+F^{\prime \prime}(\bar{\varphi}) \psi+R_{1}^{h}(\varphi-\bar{\varphi})^{2}\right)(s), A^{-1} \psi(s)\right\rangle_{V} \mathrm{~d} s\right| \\
& \leq C_{14} \int_{0}^{t}\left(\|\psi(s)\|_{H}^{2}+\|\psi(s)\|_{V^{\prime}}^{2}\right) \mathrm{d} s+C_{15}\|h\|_{L^{2}(0, t ; H)}^{4} .
\end{aligned}
$$

Next, we estimate $I_{3}$, where we discuss each of the four terms occurring in the definition of $Q^{h}$ (cf. (3.37)) individually. In the following, we repeatedly omit the time argument inside the integrals for the sake of a shorter exposition. At first, we have for every $\gamma>0$ (to be chosen later) that

$$
\begin{aligned}
& \left|\int_{0}^{t}\left\langle P(\bar{\varphi})(\chi-\zeta), A^{-1}(\psi-\chi)\right\rangle_{V} \mathrm{~d} s\right| \\
& \leq C_{16} \int_{0}^{t}\left(\|\chi\|_{V^{\prime}}+\|\zeta\|_{V^{\prime}}\right)\left(\|\psi\|_{V^{\prime}}+\|\chi\|_{V^{\prime}}\right) \mathrm{d} s \\
& \leq \gamma \int_{0}^{t}\|\zeta\|_{V^{\prime}}^{2} \mathrm{~d} s+\frac{C_{17}}{\gamma} \int_{0}^{t}\left(\|\chi\|_{V^{\prime}}^{2}+\|\psi\|_{V^{\prime}}^{2}\right) \mathrm{d} s,
\end{aligned}
$$

where in the first inequality we have used (2.17) and the fact that (recall (1.12))

$$
\|P(\bar{\varphi})(\chi-\zeta)\|_{V^{\prime}} \leq\|P(\bar{\varphi})\|_{W^{1, \infty}(\Omega)}\left(\|\chi\|_{V^{\prime}}+\|\zeta\|_{V^{\prime}}\right) \quad \text { a. e. in }(0, T) .
$$


Next, in view of (3.41), we find that

$$
\begin{aligned}
& \left|\int_{0}^{t}\left\langle\left(P\left(\varphi^{h}\right)-P(\bar{\varphi})\right)\left[\left(\sigma^{h}-\bar{\sigma}\right)-\left(\mu^{h}-\bar{\mu}\right)\right], A^{-1}(\psi-\chi)\right\rangle_{V} d s\right| \\
& \leq C_{18} \int_{0}^{t}\left\|\left(P\left(\varphi^{h}\right)-P(\bar{\varphi})\right)\left[\left(\sigma^{h}-\bar{\sigma}\right)-\left(\mu^{h}-\bar{\mu}\right)\right]\right\|_{H}\left(\|\psi\|_{V^{\prime}}+\|\chi\|_{V^{\prime}}\right) \mathrm{d} s \\
& \leq C_{19}\|h\|_{L^{2}(0, t ; H)}^{4}+C_{20} \int_{0}^{t}\left(\|\psi\|_{V^{\prime}}^{2}+\|\chi\|_{V^{\prime}}^{2}\right) \mathrm{d} s .
\end{aligned}
$$

In addition, (3.43) yields that

$$
\begin{aligned}
& \left|\int_{0}^{t}\left\langle P^{\prime}(\bar{\varphi})(\bar{\sigma}-\bar{\mu}) \psi, A^{-1}(\psi-\chi)\right\rangle_{V} \mathrm{~d} s\right| \\
& \leq C_{21} \int_{0}^{t}\left\|P^{\prime}(\bar{\varphi})(\bar{\sigma}-\bar{\mu}) \psi\right\|_{H}\left(\|\psi\|_{V^{\prime}}+\|\chi\|_{V^{\prime}}\right) \mathrm{d} s \\
& \leq \gamma \int_{0}^{t}\|\psi\|_{V}^{2} \mathrm{~d} s+\frac{C_{22}}{\gamma} \int_{0}^{t}\left(\|\psi\|_{V^{\prime}}^{2}+\|\chi\|_{V^{\prime}}^{2}\right) \mathrm{d} s .
\end{aligned}
$$

Finally, we obtain from (3.42) that

$$
\begin{aligned}
& \left|\int_{0}^{t}\left\langle(\bar{\sigma}-\bar{\mu}) R_{2}^{h}\left(\varphi^{h}-\bar{\varphi}\right)^{2}, A^{-1}(\psi-\chi)\right\rangle_{V} \mathrm{~d} s\right| \\
& \leq C_{23} \int_{0}^{t}\left\|(\bar{\sigma}-\bar{\mu}) R_{2}^{h}\left(\varphi^{h}-\bar{\varphi}\right)^{2}\right\|_{H}\left(\|\psi\|_{V^{\prime}}+\|\chi\|_{V^{\prime}}\right) \mathrm{d} s \\
& \leq C_{24}\|h\|_{L^{2}(0, t ; H)}^{4}+C_{25} \int_{0}^{t}\left(\|\psi\|_{V^{\prime}}^{2}+\|\chi\|_{V^{\prime}}^{2}\right) \mathrm{d} s .
\end{aligned}
$$

Combining the estimates (3.45) -(3.51), we have shown that for every $t \in[0, T]$ and $\gamma>0$ it holds that

$$
\begin{aligned}
& \frac{1}{2}\left(\|\psi(t)\|_{V^{\prime}}^{2}+\|\chi(t)\|_{V^{\prime}}^{2}\right)+(1-\gamma) \int_{0}^{t}\|\psi(s)\|_{V^{\prime}}^{2} \mathrm{~d} s+\int_{0}^{t} \int_{\Omega}|\chi|^{2} \mathrm{~d} x \mathrm{~d} s \\
& \leq C_{26}\|h\|_{L^{2}(0, t ; H)}^{4}+C_{27}\left(1+\gamma^{-1}\right) \int_{0}^{t}\left(\|\psi(s)\|_{V^{\prime}}^{2}+\|\chi(s)\|_{V^{\prime}}^{2}\right) \mathrm{d} s \\
& \quad+C_{28}\|\psi\|_{L^{2}(0, t ; H)}^{2}+\gamma \int_{0}^{t}\|\zeta(s)\|_{V^{\prime}}^{2} \mathrm{~d} s .
\end{aligned}
$$

We still need to control the last two terms on the right-hand side of (3.52). At first, 
notice that from the second equation in (3.44) and (3.40) we can infer that

$$
\begin{aligned}
& \int_{0}^{t}\|\zeta(s)\|_{V^{\prime}}^{2} \mathrm{~d} s \leq C_{29} \int_{0}^{t}\left(\|\psi(s)\|_{V}^{2}+\|\psi(s)\|_{V^{\prime}}^{2}\right) \mathrm{d} s \\
& \quad+C_{30} \int_{0}^{t}\left\|R_{1}^{h}(s)\left(\varphi^{h}(s)-\bar{\varphi}(s)\right)^{2}\right\|_{V^{\prime}}^{2} \mathrm{~d} s \\
& \leq C_{31} \int_{0}^{t}\left(\|\psi\|_{V}^{2}+\|\psi\|_{V^{\prime}}^{2}\right) \mathrm{d} s+C_{32}\|h\|_{L^{2}(0, t ; H)}^{4} .
\end{aligned}
$$

Now observe that the compactness of the embeddings $V \subset H \subset V^{\prime}$ implies that for every $\gamma>0$ there is some constant $C_{\gamma}>0$ such that

$$
\|v\|_{H}^{2} \leq \gamma\|v\|_{V}^{2}+C_{\gamma}\|v\|_{V^{\prime}}^{2} \quad \forall v \in V
$$

Hence, adjusting $\gamma>0$ appropriately small, invoking the estimates (3.52)-(3.54), and applying Gronwall's lemma, we can finally infer that

$$
\begin{aligned}
& \left\|\psi^{h}\right\|_{L^{\infty}\left(0, t ; V^{\prime}\right) \cap L^{2}(0, t ; V)}^{2}+\left\|\chi^{h}\right\|_{L^{\infty}\left(0, t ; V^{\prime}\right) \cap L^{2}(0, t ; H)}^{2}+\left\|\zeta^{h}\right\|_{L^{2}\left(0, t ; V^{\prime}\right)}^{2} \\
& \leq C_{33}\|h\|_{L^{2}(0, t ; H)}^{4} \quad \forall t \in[0, T] .
\end{aligned}
$$

SECOnd estimate: At first, we observe that (1.12), (3.55) and (H4) imply that

$$
\int_{0}^{t}\|P(\bar{\varphi}(s))(\chi(s)-\zeta(s))\|_{V^{\prime}}^{2} \mathrm{~d} s \leq C_{34}\|h\|_{L^{2}(0, t ; H)}^{4} \quad \forall t \in[0, T] .
$$

Hence, it follows from (3.37), (3.41)-(3.43), and (3.55) that

$$
\left\|Q^{h}\right\|_{L^{2}\left(0, t ; V^{\prime}\right)}^{2} \leq C_{35}\|h\|_{L^{2}(0, t ; H)}^{4} \quad \forall t \in[0, T] .
$$

Hence, testing (3.33) by $\chi^{h}$, we obtain, for every $t \in[0, T]$,

$$
\begin{aligned}
& \frac{1}{2}\left\|\chi^{h}(t)\right\|_{H}^{2}+\int_{0}^{t} \int_{\Omega}\left|\nabla \chi^{h}\right|^{2} \mathrm{~d} x \mathrm{~d} s=-\int_{0}^{t} \int_{\Omega} Q^{h} \chi^{h} \mathrm{~d} x \mathrm{~d} s \\
& \leq \int_{0}^{t}\left\|Q^{h}(s)\right\|_{V^{\prime}}\left\|\chi^{h}(s)\right\|_{V} \mathrm{~d} s \leq \frac{1}{2} \int_{0}^{t}\left\|\chi^{h}(s)\right\|_{V}^{2} \mathrm{~d} s+\frac{1}{2} \int_{0}^{t}\left\|Q^{h}(s)\right\|_{V^{\prime}}^{2} \mathrm{~d} s,
\end{aligned}
$$

and Gronwall's lemma shows that

$$
\left\|\chi^{h}\right\|_{L^{\infty}(0, t ; H) \cap L^{2}(0, t ; V)}^{2} \leq C_{36}\|h\|_{L^{2}(0, t ; H)}^{4} \quad \forall t \in[0, T]
$$

Third estimate: We now multiply (3.32) by $\Delta \psi$ and take $v=\psi$ in (3.31), and integrate. Adding the resulting identities, applying integration by parts and Young's 
inequality, and invoking the estimates (3.40), (3.55), and (3.57), we easily obtain that, for every $t \in[0, T]$,

$$
\begin{aligned}
& \frac{1}{2}\|\psi(t)\|_{H}^{2}+\int_{0}^{t} \int_{\Omega}|\Delta \psi|^{2} \mathrm{~d} x \mathrm{~d} s \\
& \quad \leq \int_{0}^{t} \int_{\Omega}|\Delta \psi|\left(\left|F^{\prime \prime}(\bar{\varphi}) \psi\right|+\left|R_{1}^{h}\left(\varphi^{h}-\bar{\varphi}\right)^{2}\right|\right) \mathrm{d} x \mathrm{~d} s+\int_{0}^{t}\left\|Q^{h}(s)\right\|_{V^{\prime}}\|\psi(s)\|_{V} \mathrm{~d} s \\
& \quad \leq \frac{1}{2} \int_{0}^{t} \int_{\Omega}|\Delta \psi|^{2} \mathrm{~d} x \mathrm{~d} s+C_{37}\|h\|_{L^{2}(0, t ; H)}^{4} .
\end{aligned}
$$

Using this and (3.32), we have thus shown the estimate

$$
\left\|\psi^{h}\right\|_{L^{\infty}(0, t ; H) \cap L^{2}\left(0, t ; H^{2}(\Omega)\right)}^{2}+\left\|\zeta^{h}\right\|_{L^{2}(0, t ; H)}^{2} \leq C_{38}\|h\|_{L^{2}(0, t ; H)}^{4} \quad \forall t \in[0, T] .
$$

Comparison in (3.31) then yields that also

$$
\left\|\partial_{t} \psi^{h}\right\|_{L^{2}\left(0, t ; W^{\prime}\right)}^{2} \leq C_{39}\|h\|_{L^{2}(0, t ; H)}^{4} \quad \forall t \in[0, T] .
$$

Fourth ESTIMATE: Now that (3.61) is shown, we also have

$$
\int_{0}^{t}\|P(\bar{\varphi}(s))(\chi(s)-\zeta(s))\|_{H}^{2} \mathrm{~d} s \leq C_{40}\|h\|_{L^{2}(0, t ; H)}^{4} \quad \forall t \in[0, T],
$$

which, together with (3.41)-(3.43), implies the bound

$$
\left\|Q^{h}\right\|_{L^{2}(0, t ; H)}^{2} \leq C_{41}\|h\|_{L^{2}(0, t ; H)}^{4} \quad \forall t \in[0, T] .
$$

It is then an easy task (test (3.33) first by $\partial_{t} \chi^{h}$ and then by $-\Delta \chi^{h}$ ) to see that also

$$
\left\|\chi^{h}\right\|_{H^{1}(0, t ; H) \cap L^{2}\left(0, t ; H^{2}(\Omega)\right)}^{2} \leq C_{42}\|h\|_{L^{2}(0, t ; H)}^{4} \quad \forall t \in[0, T] .
$$

With this, the inequality (3.30) is shown if we choose the function $Z(\lambda)$ as an appropriate multiple of $\lambda^{4}$. The assertion is thus proved.

REMARK 4 Since the embedding of $H^{1}\left(0, T ; W^{\prime}\right) \cap L^{2}(0, T ; W)$ into $C^{0}([0, T] ; H)$ is continuous, we infer from Theorem 3.2 that the control-to-state mapping $\mathcal{S}$ is also Fréchet differentiable into $C^{0}([0, T] ; H)$ with respect to the first variable. From this it follows that the reduced cost functional $\widetilde{\mathcal{J}}(u):=\mathcal{J}\left(\mathcal{S}_{1}(u), u\right.$ ) (where $\mathcal{S}_{1}(u)$ denotes the first component of $\mathcal{S}(u))$ is Fréchet differentiable in $\mathcal{U}_{R}$. Recalling that $\mathcal{U}_{\text {ad }}$ is a closed and convex subset of $L^{2}(Q)$, we conclude from standard arguments (which need no repetition here) the following result.

Corollary 3.3 Suppose that the assumptions $(\mathbf{H 1})-(\mathbf{H} \mathbf{6})$ are fulfilled, and assume that $\bar{u} \in \mathcal{U}_{\mathrm{ad}}$ is an optimal control for the problem (CP) with associated state 
$(\bar{\varphi}, \bar{\mu}, \bar{\sigma})=\mathcal{S}(\bar{u})$. Then we have

$$
\begin{aligned}
& \beta_{Q} \int_{0}^{T} \int_{\Omega}\left(\bar{\varphi}-\varphi_{Q}\right) \xi \mathrm{d} x \mathrm{~d} t+\beta_{\Omega} \int_{\Omega}\left(\bar{\varphi}(T)-\varphi_{\Omega}\right) \xi(T) \mathrm{d} x \\
& +\beta_{u} \int_{0}^{T} \int_{\Omega} \bar{u}(v-\bar{u}) \mathrm{d} x \mathrm{~d} x \geq 0 \quad \forall v \in \mathcal{U}_{\mathrm{ad}},
\end{aligned}
$$

where $\xi$ is the first component of the solution to the linearized system (3.1)-(3.5) for $h=v-\bar{u}$.

\section{The control problem}

Existence. Consider the control problem $(\mathbf{C P})$. We begin with the following existence result.

THEOREM 4.1 Suppose that the assumptions $(\mathbf{H 1})-(\mathbf{H} 6)$ are fulfilled. Then the optimal control problem $(\mathbf{C P})$ has a solution $\bar{u} \in \mathcal{U}_{\mathrm{ad}}$.

Proof: Let $\left\{u_{n}\right\} \subset \mathcal{U}_{\text {ad }}$ be a minimizing sequence for $(\mathbf{C P})$, and let $\left(\varphi_{n}, \mu_{n}, \sigma_{n}\right)=$ $\mathcal{S}\left(u_{n}\right), n \in \mathbb{N}$. Then it follows from (2.9) and (2.17) that there exist $(\varphi, \mu, \sigma)$ and $u \in \mathcal{U}_{\text {ad }}$ such that, possibly for a subsequence which is again indexed by $n$, we have

$$
\begin{aligned}
& u_{n} \rightarrow u \quad \text { weakly star in } L^{\infty}(Q), \\
& \varphi_{n} \rightarrow \varphi \quad \text { weakly star in } H^{1}(0, T ; V) \cap L^{\infty}\left(0, T ; H^{3}(\Omega)\right), \\
& \Delta \varphi_{n} \rightarrow \Delta \varphi \quad \text { weakly in } L^{2}(0, T ; W), \\
& \mu_{n} \rightarrow \mu \quad \text { weakly star in } L^{\infty}(0, T ; V) \cap L^{2}(0, T ; W), \\
& \sigma_{n} \rightarrow \sigma \quad \text { weakly star in } H^{1}(0, T ; H) \cap L^{\infty}(0, T ; V) \cap L^{2}(0, T ; W) .
\end{aligned}
$$

In addition, by virtue of standard compactness results (cf., e.g., [39, Sec. 8, Cor. 4]), we have the strong convergence

$$
\varphi_{n} \rightarrow \varphi \quad \text { strongly in } C^{0}\left([0, T] ; H^{2}(\Omega)\right),
$$

which implies, in particular, that

$$
\varphi_{n} \rightarrow \varphi \text { strongly in } C^{0}(\bar{Q}),
$$

whence also

$$
F^{\prime}\left(\varphi_{n}\right) \rightarrow F^{\prime}(\varphi) \text { and } P\left(\varphi_{n}\right) \rightarrow P(\varphi), \quad \text { both strongly in } C^{0}(\bar{Q}) \text {. }
$$

In summary, we can pass to the limit as $n \rightarrow \infty$ in (1.3)-(1.7), written for $\left(\varphi_{n}, \mu_{n}, \sigma_{n}\right)$, finding that $(\varphi, \mu, \sigma)=\mathcal{S}(u)$; i.e., the pair $((\varphi, \mu, \sigma), u)$ is admissible for (CP). It then follows from the weak sequential lower semicontinuity properties of $\mathcal{J}$ that $((\varphi, \mu, \sigma), u)$ is an optimal pair for $(\mathbf{C P})$. 
The adjoint system and first order necessary optimality conditions. In order to establish the necessary first order optimality conditions for (CP), we need to eliminate $\xi$ from inequality (3.66). To this end, we introduce the adjoint system which formally reads as follows:

$$
\begin{aligned}
& -\partial_{t} p+\Delta q-F^{\prime \prime}(\bar{\varphi}) q+P^{\prime}(\bar{\varphi})(\bar{\sigma}-\bar{\mu})(r-p)=\beta_{Q}\left(\bar{\varphi}-\varphi_{Q}\right) \text { in } Q, \\
& q-\Delta p+P(\bar{\varphi})(p-r)=0 \quad \text { in } Q, \\
& -\partial_{t} r-\Delta r+P(\bar{\varphi})(r-p)=0 \quad \text { in } Q, \\
& \partial_{\mathbf{n}} p=\partial_{\mathbf{n}} q=\partial_{\mathbf{n}} r=0 \quad \text { on } \Sigma, \\
& r(T)=0, \quad p(T)=\beta_{\Omega}\left(\bar{\varphi}(T)-\varphi_{\Omega}\right) \quad \text { in } \Omega .
\end{aligned}
$$

Since the final value $p(T)$ only belongs to $L^{2}(\Omega)$, we can at best expect the regularity

$$
p \in H^{1}\left(0, T ; W^{\prime}\right) \cap C^{0}([0, T] ; H) \cap L^{2}(0, T ; W),
$$

which entails that (4.1) has to be understood in a weak variational sense. More precisely, we call $(p, q, r)$ a solution to the adjoint system (4.1)-(4.5) if and only if the functions $(p, q, r)$ satisfy the following conditions:

$$
\begin{aligned}
& p \in H^{1}\left(0, T ; W^{\prime}\right) \cap C^{0}([0, T] ; H) \cap L^{2}(0, T ; W), \quad q \in L^{2}(Q), \\
& r \in H^{1}(0, T ; H) \cap C^{0}([0, T] ; V) \cap L^{2}(0, T ; W),
\end{aligned}
$$

the equations (4.1)-(4.5) are satisfied almost everywhere in their respective domains, but (4.1) and the related boundary condition in (4.4) hold true in the sense that

$$
\begin{aligned}
& \left\langle-\partial_{t} p(t), v\right\rangle_{W}+\int_{\Omega} q(t) \Delta v \mathrm{~d} x-\int_{\Omega} F^{\prime \prime}(\bar{\varphi}(t)) q(t) v \mathrm{~d} x \\
& +\int_{\Omega} P^{\prime}(\bar{\varphi}(t))(\bar{\sigma}(t)-\bar{\mu}(t))(r(t)-p(t)) v \mathrm{~d} x=\int_{\Omega} \beta_{Q}\left(\bar{\varphi}(t)-\varphi_{Q}(t)\right) v \mathrm{~d} x
\end{aligned}
$$

for all $v \in W$ and almost every $t \in(0, T)$.

We have the following existence and uniqueness result.

TheOREM 4.2 Assume that the hypotheses (H1)-(H6) hold. Then the adjoint system (4.1)-(4.5) has a unique solution in the sense formulated above.

ProOF: As in the proof of Theorem 3.1, we apply a Faedo-Galerkin approximation using the family $\left\{w_{j}\right\}_{j \in \mathbb{N}} \subset W$ as a Galerkin basis in $W$ and $\mathcal{W}_{n}$ as approximating finite-dimensional spaces. Let $n \in \mathbb{N}$ be fixed. We look for functions of the form

$$
p_{n}(x, t):=\sum_{k=1}^{n} a_{k}^{n}(t) w_{k}(x), \quad r_{n}(x, t):=\sum_{k=1}^{n} b_{k}^{n}(t) w_{k}(x), \quad q_{n}(x, t):=\sum_{k=1}^{n} c_{k}^{n}(t) w_{k}(x),
$$


which satisfy for almost every $t \in(0, T)$ the following approximating problem:

$$
\begin{aligned}
& \left(-\partial_{t} p_{n}(t), v\right)_{H}+\left(q_{n}(t), \Delta v\right)_{H}+\left(P^{\prime}(\bar{\varphi}(t))(\bar{\sigma}(t)-\bar{\mu}(t))\left(r_{n}(t)-p_{n}(t)\right), v\right)_{H} \\
& -\left(F^{\prime \prime}(\bar{\varphi}(t)) q_{n}(t), v\right)_{H}=\left(\beta_{Q}\left(\bar{\varphi}(t)-\varphi_{Q}(t)\right), v\right)_{H} \quad \forall v \in \mathcal{W}_{n}, \\
& \left(q_{n}(t), v\right)_{H}=\left(\Delta p_{n}(t)+P(\bar{\varphi}(t))\left(r_{n}(t)-p_{n}(t)\right), v\right)_{H} \quad \forall v \in \mathcal{W}_{n}, \\
& \left(-\partial_{t} r_{n}(t), v\right)_{H}+\left(-\Delta r_{n}(t)+P(\bar{\varphi}(t))\left(r_{n}(t)-p_{n}(t)\right), v\right)_{H}=0 \quad \forall v \in \mathcal{W}_{n}, \\
& r_{n}(T)=0, \quad p_{n}(T)=\mathbb{P}_{n}\left(\beta_{\Omega}\left(\bar{\varphi}(T)-\varphi_{\Omega}\right)\right),
\end{aligned}
$$

where $\mathbb{P}_{n}$ denotes the orthogonal projector in $H$ onto $\mathcal{W}_{n}$.

Arguing as in the proof of Theorem 3.1, we can again infer that the backwardin-time initial value problem (4.8)-(4.11) has a unique solution triple $\left(p_{n}, q_{n}, r_{n}\right) \in$ $\left(W^{1, \infty}\left(0, T ; \mathcal{W}_{n}\right)\right)^{3}$.

We now aim to derive a number of a priori estimates for the approximations. To this end, we denote by $C_{i}, i \in \mathbb{N}$, positive constants that may depend on the data of the problem, but not on $n \in \mathbb{N}$.

A PRIORI ESTIMATES: Let $t \in[0, T]$ be arbitrary. We insert $v=p_{n}(t)$ in (4.8), $v=-\Delta p_{n}(t) \in \mathcal{W}_{n}$ in (4.9), and $v=r_{n}(t)$ in (4.10), add the resulting equations and integrate over $[t, T]$. In view of (4.11), we find the identity

$$
\begin{aligned}
& \frac{1}{2}\left(\left\|p_{n}(t)\right\|_{H}^{2}+\left\|r_{n}(t)\right\|_{H}^{2}\right)+\int_{t}^{T} \int_{\Omega}\left(\left|\Delta p_{n}\right|^{2}+\left|\nabla r_{n}\right|^{2}\right) \mathrm{d} x \mathrm{~d} s \\
& =\frac{1}{2}\left\|\mathbb{P}_{n}\left(\beta_{\Omega}\left(\bar{\varphi}(T)-\varphi_{\Omega}\right)\right)\right\|_{H}^{2}+\int_{t}^{T} \int_{\Omega} F^{\prime \prime}(\bar{\varphi}) p_{n} q_{n} \mathrm{~d} x \mathrm{~d} s \\
& \quad+\int_{t}^{T} \int_{\Omega} \beta_{Q}\left(\bar{\varphi}-\varphi_{Q}\right) p_{n} \mathrm{~d} x \mathrm{~d} s+\int_{t}^{T} \int_{\Omega} P(\bar{\varphi})\left(p_{n}-r_{n}\right)\left(r_{n}+\Delta p_{n}\right) \mathrm{d} x \mathrm{~d} s \\
& \quad-\int_{t}^{T} \int_{\Omega} P^{\prime}(\bar{\varphi})(\bar{\sigma}-\bar{\mu})\left(r_{n}-p_{n}\right) p_{n} \mathrm{~d} x .
\end{aligned}
$$

Using Young's inequality, it is easily seen that the first four summands on the righthand side are bounded by an expression of the form

$$
C_{1}+\frac{1}{2} \int_{t}^{T} \int_{\Omega}\left|\Delta p_{n}\right|^{2} \mathrm{~d} x \mathrm{~d} s+C_{2} \int_{t}^{T} \int_{\Omega}\left(p_{n}^{2}+r_{n}^{2}\right) \mathrm{d} x \mathrm{~d} s
$$

while for the last one (which we denote by $I$ ) it follows from Hölder's inequality, (2.17) and the continuous embedding $V \subset L^{3}(\Omega)$ that, for any $\gamma>0$,

$$
\begin{aligned}
|I| & \leq C_{3} \int_{t}^{T}\left(\|\bar{\sigma}(s)\|_{6}+\|\bar{\mu}(s)\|_{6}\right)\left\|p_{n}(s)\right\|_{2}\left(\left\|p_{n}(s)\right\|_{3}+\left\|r_{n}(s)\right\|_{3}\right) \mathrm{d} s \\
& \leq \gamma \int_{t}^{T}\left(\left\|p_{n}(s)\right\|_{V}^{2}+\left\|r_{n}(s)\right\|_{V}^{2}\right) \mathrm{d} s+\frac{C_{4}}{\gamma} \int_{t}^{T} \int_{\Omega} p_{n}^{2} \mathrm{~d} x \mathrm{~d} s .
\end{aligned}
$$


Hence, applying standard elliptic estimates to $p_{n}$ and adjusting $\gamma>0$ appropriately small, we deduce from Gronwall's lemma backward in time that

$$
\left\|p_{n}\right\|_{L^{\infty}(0, T ; H) \cap L^{2}\left(0, T ; H^{2}(\Omega)\right)}+\left\|r_{n}\right\|_{L^{\infty}(0, T ; H) \cap L^{2}(0, T ; V)} \leq C_{5}
$$

Next, taking $v=q_{n}(t)$ in (4.9) and integrating in time, by (4.15) it is straightforward to deduce that

$$
\left\|q_{n}\right\|_{L^{2}(0, T ; H)} \leq C_{6}
$$

Moreover, it is an easy task (by first inserting $v=-\partial_{t} r_{n}(t)$ and then $v=-\Delta r_{n}(t)$ in (4.10) ) to show that also

$$
\left\|r_{n}\right\|_{H^{1}(0, T ; H) \cap L^{\infty}(0, T ; V) \cap L^{2}\left(0, T ; H^{2}(\Omega)\right)} \leq C_{7} .
$$

CONCLUSION OF THE PROOF: It follows from the a priori estimates that there are functions $(p, q, r)$ such that, possibly only for some subsequence which is again indexed by $n$,

$$
\begin{aligned}
& p_{n} \rightarrow p \quad \text { weakly in } L^{2}(0, T ; W), \\
& q_{n} \rightarrow q \quad \text { weakly in } L^{2}(Q), \\
& r_{n} \rightarrow r \quad \text { weakly in } H^{1}(0, T ; H) \cap L^{2}(0, T ; W),
\end{aligned}
$$

and, by continuous embedding, also

$$
r_{n} \rightarrow r \quad \text { weakly in } C^{0}([0, T] ; V) .
$$

It is now a standard matter (cf. the conclusion of the proof of Theorem 3.1) to show that the triple $(p, q, r)$ is in fact a solution to the linear system (4.1)-(4.5) having the asserted properties. Also the uniqueness can easily be proved; we can allow ourselves to leave the argument to the interested reader.

We are now in the position to eliminate $\xi$ from (3.66). We have the following result.

TheOREm 4.3 Assume that the hypotheses (H1)-(H6) are fulfilled, and suppose that $\bar{u} \in \mathcal{U}_{\mathrm{ad}}$ is an optimal control for problem $(\mathbf{C P})$ with associated state $(\bar{\varphi}, \bar{\mu}, \bar{\sigma})=$ $\mathcal{S}(\bar{u})$ and adjoint state $(p, q, r)$. Then we have

$$
\int_{0}^{T} \int_{\Omega}\left(r+\beta_{u} \bar{u}\right)(v-\bar{u}) \mathrm{d} x \mathrm{~d} t \geq 0 \quad \forall v \in \mathcal{U}_{\mathrm{ad}} .
$$

Proof: We have, owing to (3.2)-(3.4), (3.7), (4.2)-(4.5), and (4.7), the following 
identities:

$$
\begin{aligned}
0= & \int_{0}^{T} \int_{\Omega} q\left[\eta+\Delta \xi-F^{\prime \prime}(\bar{\varphi}) \xi\right] \mathrm{d} x \mathrm{~d} t=\int_{0}^{T} \int_{\Omega}\left(q \eta-F^{\prime \prime}(\bar{\varphi}) q \xi\right) \mathrm{d} x \mathrm{~d} t \\
& +\int_{0}^{T}\left\langle\partial_{t} p(t), \xi(t)\right\rangle_{W} \mathrm{~d} t+\int_{0}^{T} \int_{\Omega} F^{\prime \prime}(\bar{\varphi}) q \xi \mathrm{d} x \mathrm{~d} t \\
& -\int_{0}^{T} \int_{\Omega} P^{\prime}(\bar{\varphi})(\bar{\sigma}-\bar{\mu}) \xi(r-p) \mathrm{d} x \mathrm{~d} t+\int_{0}^{T} \int_{\Omega} \beta_{Q}\left(\bar{\varphi}-\varphi_{Q}\right) \xi \mathrm{d} x \mathrm{~d} t \\
0= & \int_{0}^{T}\left\langle\partial_{t} \xi(t), p(t)\right\rangle_{V} \mathrm{~d} t+\int_{0}^{T} \int_{\Omega} \nabla \eta \cdot \nabla p \mathrm{~d} x \mathrm{~d} t-\int_{0}^{T} \int_{\Omega} P(\bar{\varphi})(\rho-\eta) p \mathrm{~d} x \mathrm{~d} t \\
- & \int_{0}^{T} \int_{\Omega} P^{\prime}(\bar{\varphi})(\bar{\sigma}-\bar{\mu}) \xi p \mathrm{~d} x \mathrm{~d} t \\
0= & \int_{0}^{T} \int_{\Omega} r\left[\partial_{t} \rho-\Delta \rho+P^{\prime}(\bar{\varphi})(\bar{\sigma}-\bar{\mu}) \xi+P(\bar{\varphi})(\rho-\eta)-h\right] \mathrm{d} x \mathrm{~d} t \\
= & \int_{0}^{T} \int_{\Omega} \rho\left[-\partial_{t} r-\Delta r+P(\bar{\varphi}) r\right] \mathrm{d} x \mathrm{~d} t-\int_{0}^{T} \int_{\Omega} r h \mathrm{~d} x \mathrm{~d} t \\
& +\int_{0}^{T} \int_{\Omega} \xi P^{\prime}(\bar{\varphi})(\bar{\sigma}-\bar{\mu}) r \mathrm{~d} x \mathrm{~d} t-\int_{0}^{T} \int_{\Omega} \eta P(\bar{\varphi}) r \mathrm{~d} x \mathrm{~d} t .
\end{aligned}
$$

Next, we employ integration by parts with respect to time in the second integral on the right-hand side of (4.19) (which is permitted since $p, \xi \in H^{1}\left(0, T ; W^{\prime}\right) \cap L^{2}(0, T ; W)$ ) to conclude that

$$
I:=\int_{0}^{T}\left\langle\partial_{t} p(t), \xi(t)\right\rangle_{W} \mathrm{~d} t=\int_{\Omega} p(T) \xi(T) \mathrm{d} x-\int_{0}^{T}\left\langle\partial_{t} \xi(t), p(t)\right\rangle_{W} \mathrm{~d} t .
$$

Now observe that $p \in L^{2}(0, T ; V)$ and $\partial_{t} \xi \in L^{2}\left(0, T ; V^{\prime}\right)$. It then follows from the second condition in (4.5) that

$$
I=\int_{\Omega} \beta_{\Omega}\left(\bar{\varphi}(T)-\varphi_{\Omega}\right) \xi(T) \mathrm{d} x-\int_{0}^{T}\left\langle\partial_{t} \xi(t), p(t)\right\rangle_{V} \mathrm{~d} t
$$

Therefore, addition of the three identities (4.19)-(4.21) results in

$$
0=\int_{0}^{T} \int_{\Omega} \beta_{Q}\left(\bar{\varphi}-\varphi_{Q}\right) \xi \mathrm{d} x \mathrm{~d} t+\int_{\Omega} \beta_{\Omega}\left(\bar{\varphi}(T)-\varphi_{\Omega}\right) \xi(T) \mathrm{d} x-\int_{0}^{T} \int_{\Omega} r h \mathrm{~d} x \mathrm{~d} t
$$

and insertion of this identity in (3.66) yields the assertion.

REMARK 5 The state system (1.3)-(1.7), written for $(\varphi, \mu, \sigma)=(\bar{\varphi}, \bar{\mu}, \bar{\sigma})$, the adjoint system and the variational inequality (4.18) together form the first-order necessary optimality conditions. Moreover, since $\mathcal{U}_{\text {ad }}$ is a nonempty, closed, and convex subset of $L^{2}(Q)$, (4.18) implies that for $\beta_{u}>0$ the optimal control $\bar{u}$ is the $L^{2}(Q)$ orthogonal projection of $-\beta_{u}^{-1} r$ onto $\mathcal{U}_{\text {ad }}$, that is, we have

$$
\bar{u}(x, t)=\max \left\{u_{\min }(x, t), \min \left\{-\beta_{u}^{-1} r(x, t), u_{\max }(x, t)\right\}\right\} \quad \text { for a. e. }(x, t) \in Q .
$$


Colli - Gilardi - Rocca - Sprekels

\section{References}

[1] R. P. Araujo, D. L. S. McElwain, A history of the study of solid tumor growth: the contribution of mathematical modelling, Bull. Math. Biol. 66 (2004), 1039-1091.

[2] C. Benosman, B. Aïnseba, A. Ducrot, Optimization of cytostatic leukemia therapy in an advection-reaction-diffusion model, J. Optim. Theory Appl. 167 (2015), $296-325$.

[3] S. Bosia, M. Conti, M. Grasselli, On the Cahn-Hilliard-Brinkman system, Comm. Math. Sci. 13 (2015), 1541-1567.

[4] H. M. Byrne, M. A. J. Chaplain, Growth of nonnecrotic tumors in the presence and absence of inhibitors, Math. Biosci. 130 (1995), 151-181.

[5] C. Chatelin, T. Balois, P. Ciarletta, M. Ben Ammar, Emergence of microstructural patterns in skin cancer: a phase separation analysis in a binary mixture, New J. Phys. 13 (2011), 115013 (21 pp.)

[6] Y. Chen, S. M. Wise, V. B. Shenoy, J. S. Lowengrub, A stable scheme for a nonlinear, multiphase tumor growth model with an elastic membrane, Int. J. Numer. Methods Biomed. Eng. 30 (2014), 726-754.

[7] P. Colli, M. H. Farshbaf-Shaker, G. Gilardi, J. Sprekels, Optimal boundary control of a viscous Cahn-Hilliard system with dynamic boundary condition and double obstacle potentials, SIAM J. Control Optim. 53 (2015), 2696-2721.

[8] P. Colli, M. H. Farshbaf-Shaker, G. Gilardi, J. Sprekels, Second-order analysis of a boundary control problem for the viscous Cahn-Hilliard equation with dynamic boundary conditions, Ann. Acad. Rom. Sci. Math. Appl. 7 (2015), 41-66.

[9] P. Colli, G. Gilardi, D. Hilhorst, On a Cahn-Hilliard type phase field system related to tumor growth, Discrete Contin. Dyn. Syst. 35 (2015), 2423-2442.

[10] P. Colli, G. Gilardi, E. Rocca, J. Sprekels, Vanishing viscosities and error estimate for a Cahn-Hilliard type phase field system related to tumor growth, Nonlinear Anal. Real World Appl. 26 (2015), 93-108.

[11] P. Colli, G. Gilardi, E. Rocca, J. Sprekels, Asymptotic analyses and error estimates for a Cahn-Hilliard type phase field system modelling tumor growth. Discrete Contin. Dyn. Syst. Ser. S, to appear (see also preprint arXiv:1503.00927 [math.AP] (2015), 1-19).

[12] P. Colli, G. Gilardi, J. Sprekels, Analysis and optimal boundary control of a nonstandard system of phase field equations, Milan J. Math. 80 (2012), 119-149.

[13] P. Colli, G. Gilardi, J. Sprekels, A boundary control problem for the viscous Cahn-Hilliard equation with dynamic boundary conditions, Appl. Math. Optim., DOI 10.1007/s00245-015-9299-z . 
[14] P. Colli, G. Gilardi, J. Sprekels, A boundary control problem for the pure CahnHilliard equation with dynamic boundary conditions, Adv. Nonlinear Anal. 4 (2015), 311-325.

[15] V. Cristini, J. Lowengrub, Multiscale modeling of cancer. An Integrated Experimental and Mathematical Modeling Approach, Cambridge University Press, Cambridge, 2010.

[16] V. Cristini, X. Li, J. S. Lowengrub, S. M. Wise, Nonlinear simulations of solid tumor growth using a mixture model: invasion and branching, J. Math. Biol. 58 (2009), 723-763.

[17] M. Dai, E. Feireisl, E. Rocca, G. Schimperna, M. Schonbek, Analysis of a diffuse interface model of multispecies tumor growth, preprint arXiv:1507.07683 [math.AP] (2015), 1-18.

[18] H. B. Frieboes, F. Jin, Y.-L. Chuang, S. M. Wise, J. S. Lowengrub, V. Cristini, Three-dimensional multispecies nonlinear tumor growth-II: Tumor invasion and angiogenesis, J. Theoret. Biol. 264 (2010), 1254-1278.

[19] S. Frigeri, M. Grasselli, E. Rocca, On a diffuse interface model of tumor growth, European J. Appl. Math. 26 (2015), 215-243.

[20] S. Frigeri, E. Rocca, J. Sprekels, Optimal distributed control of a nonlocal CahnHilliard/Navier-Stokes system in 2D, SIAM J. Control Optim., to appear (see also WIAS preprint No. 2036 (2014), 1-30).

[21] C. G. Gal, M. Grasselli, Longtime behavior of nonlocal Cahn-Hilliard equations, Discrete Contin. Dyn. Syst. 34 (2014), 145-179.

[22] H. Garcke, K.F. Lam, Well-posedness of a Cahn-Hilliard system modelling tumour growth with chemotaxis and active transport, preprint arXiv:1511.06143 [math.AP] (2015), 1-28.

[23] H. Garcke, K.F. Lam, E. Sitka, V. Styles, A Cahn-Hilliard-Darcy model for tumour growth with chemotaxis and active transport, preprint arXiv:1508.00437 [math.AP] (2015), 1-45.

[24] A. Hawkins-Daarud, S. Prudhomme, K. G. van der Zee, J. T. Oden, Bayesian calibration, validation, and uncertainty quantification of diffuse interface models of tumor growth, J. Math. Biol. 67 (2013), 1457-1485.

[25] A. Hawkins-Daruud, K. G. van der Zee, J. T. Oden, Numerical simulation of a thermodynamically consistent four-species tumor growth model, Int. J. Numer. Math. Biomed. Engng. 28 (2011), 3-24.

[26] D. Hilhorst, J. Kampmann, T. N. Nguyen, K. G. van der Zee, Formal asymptotic limit of a diffuse-interface tumor-growth model, Math. Models Methods Appl. Sci. 25 (2015), 1011-1043. 
[27] M. Hintermüller, T. Keil, D. Wegner, Optimal control of a semidiscrete Cahn-Hilliard-Navier-Stokes system with non-matched fluid densities, preprint arXiv:1506.03591 [math.AP] (2015), 1-35.

[28] M. Hintermüller, D. Wegner, Distributed optimal control of the Cahn-Hilliard system including the case of a double-obstacle homogeneous free energy density, SIAM J. Control Optim. 50 (2012), 388-418.

[29] M. Hintermüller, D. Wegner, Optimal control of a semidiscrete Cahn-HilliardNavier-Stokes system, SIAM J. Control Optim. 52 (2014), 747-772.

[30] M. Hintermüller, D. Wegner, Distributed and boundary control problems for the semidiscrete Cahn-Hilliard/Navier-Stokes system with nonsmooth GinzburgLandau energies, Isaac Newton Institute Preprint Series No. NI14042-FRB (2014), $1-29$.

[31] J. Jiang, H. Wu, S. Zheng, Well-posedness and long-time behavior of a nonautonomous Cahn-Hilliard-Darcy system with mass source modeling tumor growth, J. Differential Equations 259 (2015), 3032-3077.

[32] U. Ledzewicz, H. Schättler, Multi-input optimal control problems for combined tumor anti-angiogenic and radiotherapy treatments, J. Optim. Theory Appl. 153 (2012), 195-224.

[33] J. S. Lowengrub, H. B. Frieboes, F. Lin, Y.-L. Chuang, X. Li, P. Macklin, S. M. Wise, V. Cristini, Nonlinear modelling of cancer: bridging the gap between cells and tumors, Nonlinearity 23 (2010), R1-R91.

[34] J. S. Lowengrub, E. Titi, K. Zhao, Analysis of a mixture model of tumor growth, European J. Appl. Math. 24 (2013), 1-44.

[35] L. Nirenberg, On elliptic partial differential equations, Ann. Scuola Norm. Sup. Pisa (3) 13 (1959), 115-162.

[36] J. T. Oden, A. Hawkins, S. Prudhomme, General diffuse-interface theories and an approach to predictive tumor grwth modeling, Math. Models Methods Appl. Sci. 20 (2010), 477-517.

[37] J. T. Oden, E. E. Prudencio, A. Hawkins-Daruud, Selection and assessment of phenomenological models of tumor growth, Math. Models Methods Appl. Sci. 23 (2013), 1309-1338.

[38] E. Rocca, J. Sprekels, Optimal distributed control of a nonlocal convective CahnHilliard equation by the velocity in three dimensions, SIAM J. Control Optim. 53 (2015), 1654-1680.

[39] J. Simon, Compact sets in the space $L^{p}(0, T ; B)$, Ann. Mat. Pura Appl. (4) 146 (1987), 65-96. 
[40] Q.-F. Wang, S.-i. Nakagiri, Weak solutions of Cahn-Hilliard equations having forcing terms and optimal control problems, Mathematical models in functional equations (Japanese) (Kyoto, 1999), Sūrikaisekikenkyūsho Kōkyūroku No. 1128 (2000), 172-180.

[41] X. Wang, H. Wu, Long-time behavior for the Hele-Shaw-Cahn-Hilliard system, Asymptot. Anal. 78 (2012), 217-245.

[42] X. Wang, Z. Zhang, Well-posedness of the Hele-Shaw-Cahn-Hilliard system, Ann. Inst. H. Poincaré Anal. Non Linéaire 30 (2013), 367-384.

[43] S. M. Wise, J. S. Lowengrub, H. B. Frieboes, V. Cristini, Three-dimensional multispecies nonlinear tumor growth-I: Model and numerical method, J. Theoret. Biol. 253 (2008), 524-543.

[44] X. Wu, G. J. van Zwieten, K. G. van der Zee, Stabilized second-order splitting schemes for Cahn-Hilliard models with applications to diffuse-interface tumorgrowth models, Int. J. Numer. Meth. Biomed. Engng. 30 (2014), 180-203.

[45] X. Zhao, C. Liu, Optimal control of the convective Cahn-Hilliard equation, Appl. Anal. 92 (2013), 1028-1045.

[46] X. Zhao, C. Liu, Optimal control for the convective Cahn-Hilliard equation in 2D case, Appl. Math. Optim. 70 (2014), 61-82. 